ARTICLE

https://doi.org/10.1038/s41467-019-12368-2

\title{
Homozygous receptors for insulin and not IGF-1 accelerate intimal hyperplasia in insulin resistance and diabetes
}

Qian Li,2, Jialin Fu ${ }^{1,2}$, Yu Xia1,2,3, Weier $\mathrm{Qi}^{4}$, Atsushi Ishikado1,2, Kyoungmin Park (D) 1,2, Hisashi Yokomizo (i) ${ }^{1,2}$, Qian Huang ${ }^{2}$, Weikang Cai ${ }^{2}$, Christian Rask-Madsen ${ }^{2}$, C. Ronald Kahn² \& George L. King ${ }^{1,2 \star}$

Insulin and IGF-1 actions in vascular smooth muscle cells (VSMC) are associated with accelerated arterial intima hyperplasia and restenosis after angioplasty, especially in diabetes. To distinguish their relative roles, we delete insulin receptor (SMIRKO) or IGF-1 receptor (SMIGF1RKO) in VSMC and in mice. Here we report that intima hyperplasia is attenuated in SMIRKO mice, but not in SMIGF1RKO mice. In VSMC, deleting IGF1R increases homodimers of $I R$, enhances insulin binding, stimulates $p$-Akt and proliferation, but deleting IR decreases responses to insulin and IGF-1. Studies using chimeras of IR(extracellular domain)/IGF1R (intracellular-domain) or IGF1R(extracellular domain)/IR(intracellular-domain) demonstrate homodimer IR $\alpha$ enhances insulin binding and signaling which is inhibited by IGF1R $\alpha$. RNA-seq identifies hyaluronan synthase 2 as a target of homo-IR, with its expression increases by $I R$ activation in SMIGF1RKO mice and decreases in SMIRKO mice. Enhanced intima hyperplasia in diabetes is mainly due to insulin signaling via homo-IR, associated with increased Has2 expression.

\footnotetext{
${ }^{1}$ Dianne Nunnally Hoppes Laboratory for Diabetes Complications, Section of Vascular Cell Biology, Joslin Diabetes Center, Harvard Medical School, Boston MA 02215, USA. ${ }^{2}$ Joslin Diabetes Center, Harvard Medical School, Boston, MA 02215, USA. ${ }^{3}$ Department of Centre Laboratory, Shandong Provincial Hospital Affiliated to Shandong University, Jinan, Shandong 250001, China. ${ }^{4}$ Translational Research and Early Clinical Development, Cardiovascular and Metabolic Research, AstraZeneca, Mölndal, Sweden. *email: George.king@joslin.harvard.edu
} 
ercutaneous coronary angioplasty (PTCA) is an effective treatment for coronary artery diseases ${ }^{1}$. Although drugeluting stents have significantly reduced the risk of restenosis, it remains a major cause of stent failure ${ }^{1}$. In people with diabetes and insulin resistance, there is a significant increase in the rate of restenosis after $\mathrm{PTCA}^{2}$, which is related to the increased migration and proliferation of vascular smooth muscle cells (VSMC), as well as influx of inflammatory cells ${ }^{3,4}$.

Multiple factors have been suggested to explain the elevated risk of restenosis in association with insulin resistance and diabetes. A long-held assumption is that insulin-like growth factor-1 (IGF-1) and possibly insulin are involved, since both hormones have been reported to stimulate the migration and proliferation of VSMC $^{5-10}$. In addition, circulating insulin and IGF-1 levels are elevated in people with type 2 diabetes ${ }^{11}$. Furthermore, in several rodent models of insulin resistance and type 2 diabetes, intimal hyperplasia after vascular injury is more severe than the nondiabetic or insulin-sensitive controls ${ }^{12-14}$. However, there is a long-standing controversy on whether the accelerated intimal hyperplasia in insulin-resistant and diabetic states are due to the actions of insulin or IGF1, as well as their respective receptors ${ }^{15}$.

This lack of clarity on whether insulin or IGF-1 is the main driver of restenosis in diabetes and insulin-resistance states is due to the fact that both insulin and IGF-1 and their receptors have very similar and overlapping cellular and biological responses in VSMC and in many other cell types ${ }^{16}$. The prevailing theory on the actions of insulin and IGF-1 is that insulin binds to insulin receptors (IR) ${ }^{17,18}$ at high affinity and mediates mainly the metabolic effects of the hormone, such as those involved on the transport and the metabolism of glucose and amino acids in cells, including VSMC ${ }^{18-22}$. However, IGF-1 and IGF-1 receptors $(\mathrm{IGF} 1 \mathrm{R})^{23}$ are involved to a greater extent in the growth-related actions, including cellular migration, DNA synthesis, and possibly extracellular matrix turnover ${ }^{24-27}$. However, it is believed that IGF-1 with minor contribution from insulin enhances the growth activities in VSMC, most likely by binding to IGF-1 receptors. The assumption for a greater role for IGF-1 and IGF-1R are based on the finding that the concentration of insulin to induce VSMC proliferation is 10 to 100 times greater than its physiological levels ${ }^{6}$. Thus, it has been assumed that IGF1R are mainly responsible for the acceleration of arterial intima hyperplasia in diabetes and insulin-resistant states.

In order to determine the relative importance of IR and IGF1R in mediating the growth of VSMC to accelerate intimal hyperplasia in vivo, we have deleted IR or IGF1R from VSMC and determined their actions on VSMC proliferation and intimal hyperplasia both in culture and in vivo. The mechanism of insulin and IR's growth-promoting effects compared with IGF1R have been also characterized by analysis of IR and IGF1R structures with identification of IR's downstream target in VSMC by RNA sequencing. Surprisingly, these studies reveal that insulin acting via IR is mainly responsible for enhancing VSMC's actions to accelerate intimal hyperplasia in insulin resistance and diabetes and that this occurs by its effects on gene expression, involved in the extracellular matrix remodeling, like hyaluronan synthase.

\section{Results}

Characterization of IR deletion in VSMC of SMIRKO mice. To characterize the function of IR in VSMCs in vivo, IR were deleted in VSMC by breeding IR flox/flox mice with SM22 $\alpha$-Cre transgenic mice. These SMIRKO mice had no detectable IR in the aorta. In contrast, the expressions of IR in the brain, liver, kidney, and adipocyte were similar between SMIRKO and WT mice, although IR were partially reduced in the heart of SMIRKO mice, as expected (Supplementary Fig. 1). Insulin stimulation of Akt phosphorylation in vivo revealed an $8.0 \pm 1.8$-fold increase in $\mathrm{p}$ Akt in the aorta of WT mice, but only $2.7 \pm 0.8$-fold in SMIRKO mice (Supplementary Fig. 2). By contrast, insulin-induced p-Akt in the liver and skeletal muscle were similar in the two groups of mice (Supplementary Fig. 2). Although IR expressions were reduced partially in the myocardium of SMIRKO vs. WT mice, insulin's induction of p-Akt in the heart was similar (Supplementary Fig. 2). VSMC from WT and SMIRKO mice also exhibited selective deletion of IR in VSMC from SMIRKO, but IR levels in the endothelial cell from SMIRKO and WT mice were not different (Supplementary Fig. 1D).

Intimal hyperplasia of femoral artery induced by wire injury. The extent of intimal hyperplasia of the femoral artery after wire injury was determined in WT and SMIRKO mice, placed on a HFD for 8 weeks. Body weight, blood pressure, plasma insulin, lipid levels, intraperitoneal glucose tolerance test (IPGTT), and insulin tolerance tests (IPITT) did not differ between the two groups of mice (Supplementary Fig. 3).

The extent of intimal hyperplasia assessed by elastin staining in the femoral artery of SMIRKO mice was decreased by $37 \%$ compared with those of WT mice (Supplementary Fig. 4A, B). The ratio of intima/media of SMIRKO mice was also decreased compared with WT mice (Supplementary Fig. 4C), although the media area was not different (Supplementary Fig. 4D). VSMC proliferation, as determined by BrdU and SM22a double staining at 7 days after injury was decreased by $51 \%$ in SMIRKO mice compared with WT mice (Supplementary Fig. 4E, F). Proliferation of VSMC in the femoral artery after wire injury as assessed by cyclin A2 mRNA expression increased 3.6-fold in WT mice, which was significantly decreased by $32 \%$ in SMIRKO mice (Supplementary Fig. 4G). Similarly, cellular proliferation of VSMCs cultured from aorta showed insulin and IGF-1 increased Edu incorporation by 92 and 232\%, respectively in WT VSMCs, but only by 41 and $143 \%$ in SMIRKO mice, respectively (Supplementary Fig. 4H). IGF1, but not insulin, significantly increased proliferation of VSMCs from SMIRKO mice.

To exclude the effect of IR deletion in the heart and macrophages, IR were also knocked out by Cre recombinase driven by Myh11 promoter (Myh11IRKO). IR expression was significantly decreased in the aorta of Myh11IRKO mice compared with WT mice, whereas its expressions were not different in the heart, skeletal muscle, and liver between two groups of mice (Supplementary Fig. 5). When the mice were subjected to femoral artery injury, the intimal area and intima/media ratio were decreased significantly by 49 and $60 \%$, respectively, in the femoral artery of Myh11dIRKO mice. The thickness of arterial media was not different between two groups of mice (Fig. 1a-d). VSMC proliferation, as determined by BrdU and SM22a double staining at 7 days after injury, was significantly decreased in Myh11IRKO mice compared with WT mice (Fig. 1e, f). Cellular proliferation of cultured aortic VSMCs, determined by EdU incorporation, increased by 2.7 -fold with insulin in WT VSMCs, but only 1.5 fold in Myh11IRKO VSMCs (Fig. 1g).

Deletion of IGF1R in VSMC of SMIGF1RKO mice. SMIGF1RKO mice were generated by breeding IGF1R flox/flox mice with Myh11-Cre transgenic mice. IGF1R protein expression was decreased by $70 \%$ in the aorta of SMIGF1RKO mice compared with WT mice, which was unchanged in the heart and brain (Supplementary Fig. 6). The selectivity of IGF1R deletion in VSMC was further confirmed since no differences in IGF1R protein expressions were observed in endothelial cells from SMIGF1RKO vs. WT mice (Supplementary Fig. 6). After HFD, 
a

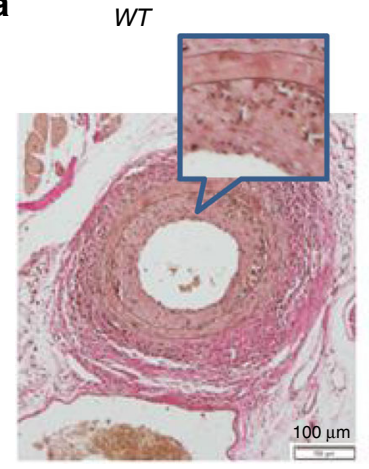

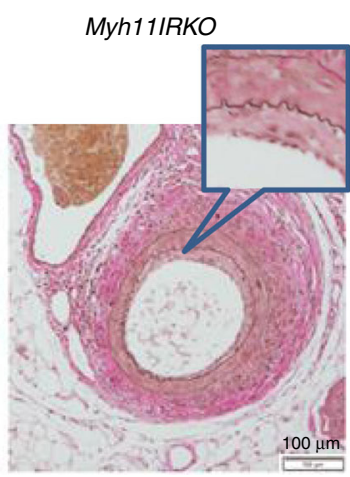

b
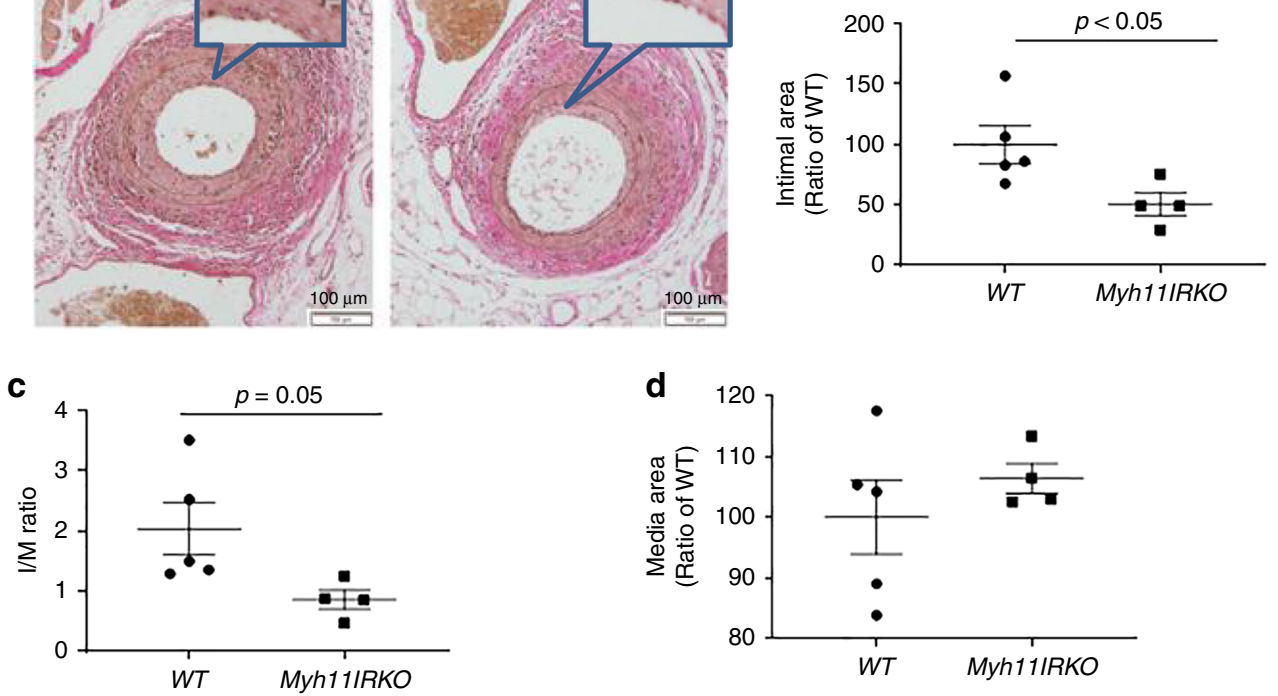

e

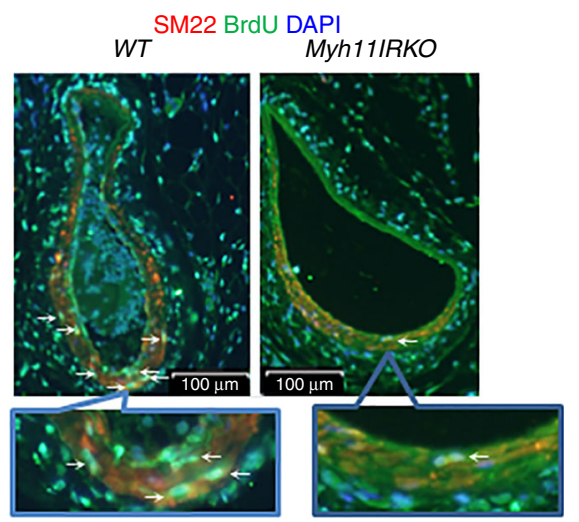

f
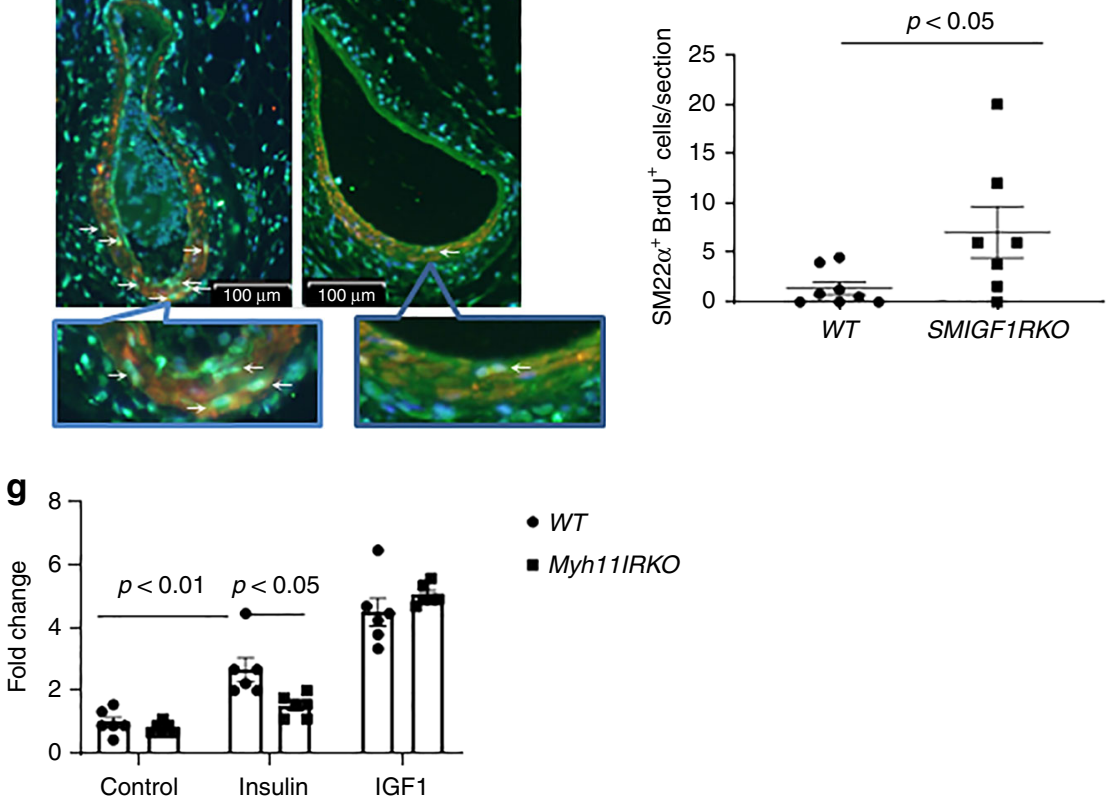

Fig. 1 Wire injury-induced femoral artery intimal hyperplasia in HFD-fed WT and Myh11IRKO mice. a-d Intimal hyperplasia was determined by elastin staining. a indicates representative images. Summarized intimal area (b), intima/media (I/M) ratio (c), and media area (d) are shown (WT, $n=5$; Myh11IRKO, $n=4)$. e, $\mathbf{f}$ VSMC proliferation in the wire-injured femoral artery. The femoral arteries were subjected to wire injury, and then the mice were infused with Brdu with osmotic minipump for 1 week. VSMCs proliferation was determined by SM22 $\alpha$ and Brdu double staining. e indicats representative images, and $\mathbf{f}$ shows summarized data (WT, $n=8 ;$ Myh11IRKO, $n=7)$. $\mathbf{g}$ Insulin and IGF- 1 induced VSMCs proliferation. Starved WT or Myh11IRKO VSMCs were stimulated with insulin $(10 \mathrm{nM})$ or IGF-1 $(10 \mathrm{nM})$ for $24 \mathrm{~h}$, and cell proliferation was determined by Edu incorporation and measured by flow cytometry ( $n=6$ per group). The data are mean \pm SEM. Two-tailed $t$ test $(\mathbf{b}, \mathbf{c}, \mathbf{d}, \mathbf{f})$ or two-way ANOVA with a post hoc test $(\mathbf{g})$. Source data are provided as a Source Data file

body weights, plasma lipid, IPGTT, and IPITT were not different between the two groups of mice (Supplementary Fig. 7A-E). Intimal area of the femoral artery was increased by $45 \%$ in SMIGF1RKO mice compared with WT mice (Fig. 2a, b). The intima/media ratio, which is the gold standard for restenosis, and media area were not different between $W T$ and SMIGF1RKO mice (Fig. 2c, d).
Sections of wire-injured femoral artery from $W T$ or SMIGF1RKO mice were stained with VSMC marker SM22 $\alpha$ or macrophages marker Mac2. VSMC numbers in the intimal area of femoral artery from SMIGF1RKO mice were increased by $50 \%$ compared with that of WT mice. The macrophages and the collagen content (trichrome staining) in the intimal area of femoral artery of WT mice were not different compared with that 
a
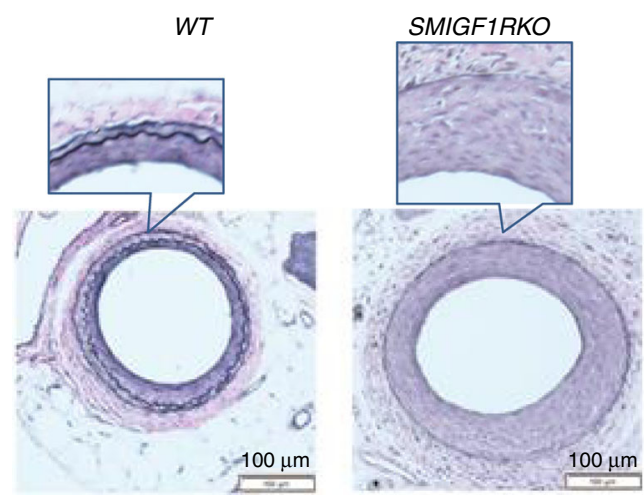

C

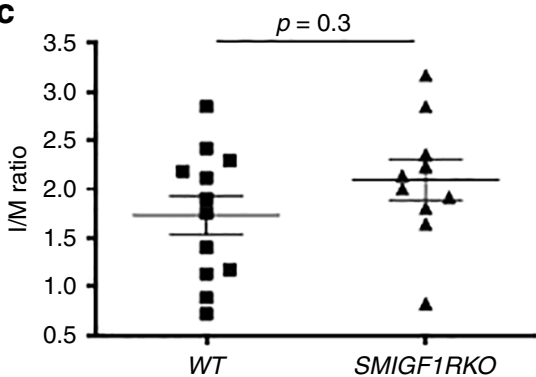

e

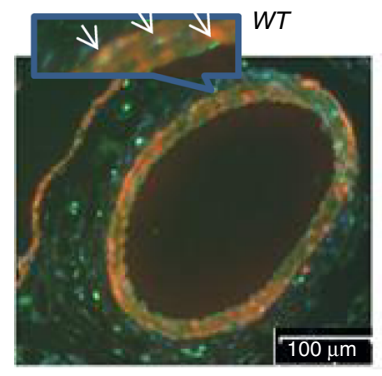

SM22 BrdU DAPI

SMIGF1RKO
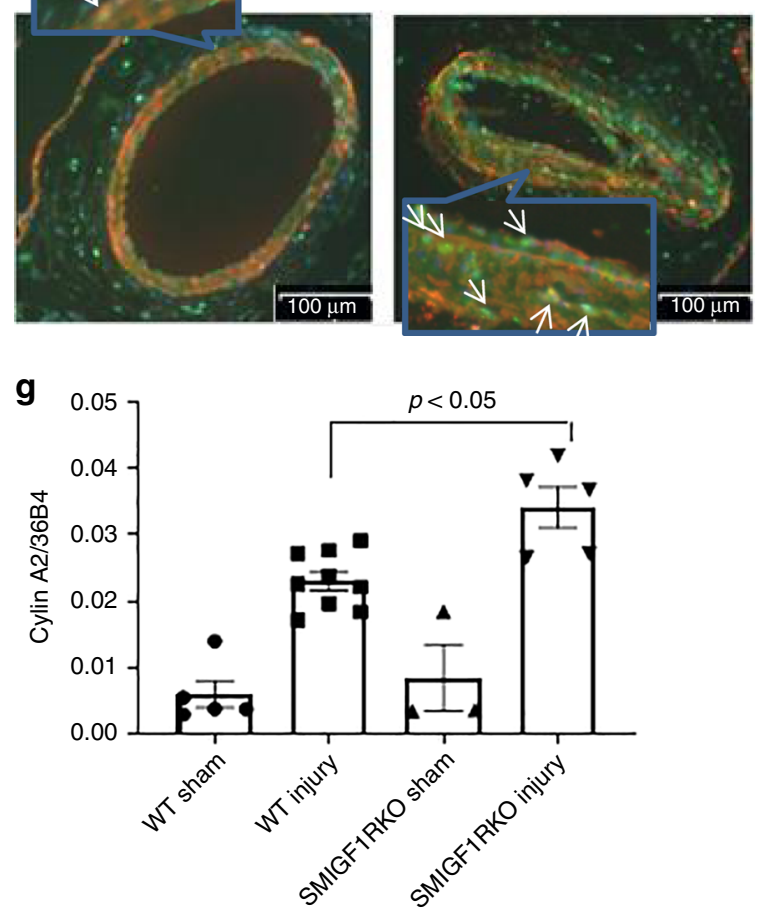

b

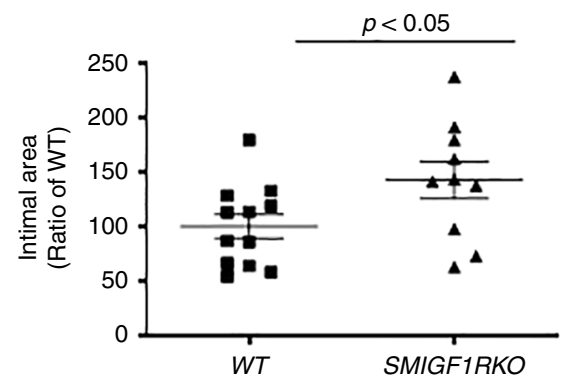

d

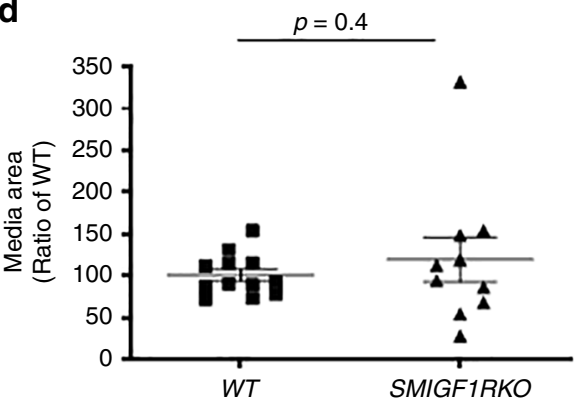

f

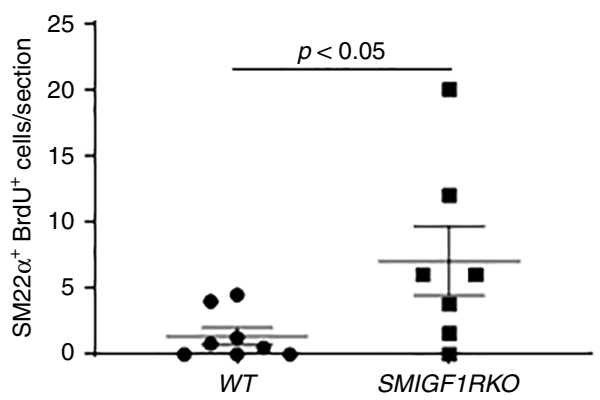

h

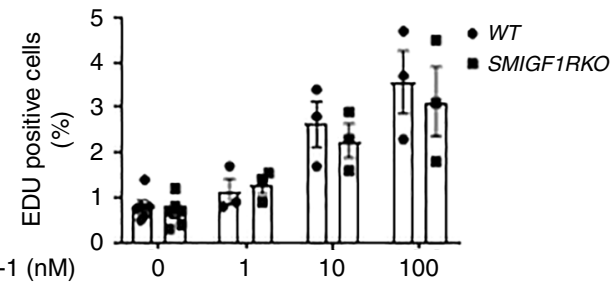

IGF-1 (nM)

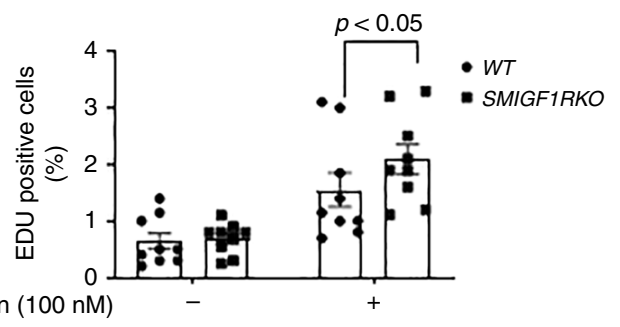

Fig. 2 Wire injury-induced femoral artery intimal hyperplasia in HFD-fed WT and SMIGF1RKO mice. a-d Intimal hyperplasia of femoral artery was determined by elastin staining at 4 weeks after wire injury. a indicates representative images. Summarized intimal area (b), intima/media (I/M) ratio (c), and media area (d) are shown (WT, $n=12$; SMIGF1RKO, $n=10$ ). E-F VSMCs proliferation. The femoral arteries were subjected to wire injury, and then the mice were infused with Brdu with osmotic minipump for 1 week. VSMCs proliferation was determined by SM22 $\alpha$ and Brdu double staining. e indicates representative images, and $\mathbf{f}$ shows summarized data $(W T, n=8$; SMIGFIRKO, $n=7)$. $\mathbf{g}$ Cyclin A2 gene expression. Cyclin A2 gene expression in femoral artery at 9 day after wire injury was used as a surrogate marker of cell proliferation, and was determined by qPCR (WT sham, $n=5 ;$ WT injury, $n=9 ;$; SMIGFIRKO sham, $n=3$; SMIGF1RKO injury, $n=5$ ). h IGF-1 and insulin-induced WT or SMIGF1RKO VSMCs proliferation. Starved VSMCs were stimulated with 1-100 nM IGF-1 or 100 $\mathrm{nM}$ insulin ( $n=9$ for each group) for $24 \mathrm{~h}$, and cell proliferation was determined by Edu incorporation and measured by flow cytometry. All data from this figure are mean \pm SEM. Two-tailed $t$ test $(\mathbf{b}, \mathbf{c}, \mathbf{d}, \mathbf{f})$ or two-way ANOVA with a post hoc test $(\mathbf{g}, \mathbf{h})$. Source data are provided as a Source Data file 
of SMIGF1RKO mice (Supplementary Fig. 8). Proliferation of VSMC, as measured by BrdU and SM22 double staining, was increased by fivefold in wire-injured femoral artery of SMIGF1RKO vs. WT mice (Fig. 2e, f), which was confirmed by increase $(48 \%)$ of cyclinA2 mRNA expression in the femoral artery of SMIGF1RKO compared with that of WT mice (Fig. $2 \mathrm{~g}$ ). Proliferation of cultured VSMC, as evaluated by Edu incorporation, showed that the effect of IGF-1 (1 to $100 \mathrm{nM}$ ) was similar in VSMC from the two types of mice (Fig. 2h). In contrast, VSMC proliferation induced by $100 \mathrm{nM}$ insulin was increased by $35 \%$ in cells from SMIGF1RKO mice compared with WT mice (Fig. 2i).

Insulin and IGF1 signaling in VSMCs. To investigate the mechanisms underlying the findings of decreased intimal hyperplasia in SMIRKO mice, but not in SMIGF1RKO mice, insulin and IGF-1's signaling in cultured VSMCs from SMIRKO, $S M I G F 1 R K O$, and $W T$ mice were studied. In cells from the SMIRKO mice, IR content was decreased $>95 \%$, without changes in IGFIR protein expression. Conversely, in VSMCs from SMIGFIRKO mice, IGF1R expression was decreased by $70 \%$ without changes in IR expressions (Fig. 3a, b).Tyrosine phosphorylations at 1150/1151 of IR (pTyr-IR) and 1135/1136 of IGF1R (pTyr IGF-IR) can be assessed using the same antibody, but the receptors can be differentiated by molecular weights, since $\operatorname{IGF} 1 \mathrm{R} \beta$ is $2 \mathrm{kDa}$ greater than $\operatorname{IR} \beta^{28,29}$ (Fig. 3a). Insulin increased pTyr-IR significantly at Y1150/1151 in WT VSMCs in a dosedependent manner reaching maximum at $100 \mathrm{nM}$. No pTyr-IR was observed in SMIRKO VSMCs even at $100 \mathrm{nM}$ insulin, although insulin increased pTyr-IGF1R (Fig. 3a). Unexpectedly, insulin-induced pTyr-IR was significantly increased to greater levels in SMIGF1RKO compared with WT VSMCs. Insulin stimulated pTyr-IR in a dose-dependent manner from $1 \mathrm{nM}$ to 100 $\mathrm{nM}$ in both WT and SMIGF1RKO mice, but the effects of insulin were more pronounced in SMIGF1RKO mice than WT mice. Levels of pTyr-IR were increased fivefold greater by insulin at $100 \mathrm{nM}$ in SMIGF1RKO VSMCs compared with WT VSMCs (Fig. 3a). In parallel with the changes in pTyr-IR, insulin increased p-Akt in a dose-dependent manner in WT VSMCs, and its effects were attenuated in SMIRKO mice by more than $60 \%$. Consistent with the elevated levels of pTyr-IR in SMIGF1RKO VSMCs, insulin-stimulated p-Akt was also significantly greater than that in WT VSMC after stimulation with $10 \mathrm{nM}$ or $100 \mathrm{nM}$ insulin (Fig. 3a). Insulin also induced p-Erk in WT VSMCs from $1 \mathrm{nM}$ to $100 \mathrm{nM}$, which was significantly lower in SMIRKO compared with WT VSMC (Fig. 3a). Interestingly, insulininduced p-Erk was not enhanced in SMIGF1RKO VSMC compared with SMIRKO VSMC. Furthermore, PDGF-induced p-Akt and p-Erk were comparable between $W T$ and SMIRKO VSMCs (Supplementary Fig. 9).

IGF-1-induced pTyr-IGF1R levels were decreased by 26 and $50 \%$ in SMIRKO VSMCs and in SMIGF1RKO VSMCs, respectively, compared with WT VSMCs (Fig. 3b). Similarly, IGF-1induced p-Akt in WT VSMCs from 1-100 nM by 2.7 to 16.5 -fold, but IGF-1-induced p-Akt was lower in SMIGF1RKO VSMCs compared with WT VSMCs (Fig. 3b). IGF-1 (100 nM)-induced pErk was also decreased by 39 and 45\%, respectively, in SMIRKO VSMCs and in SMIGF1RKO VSMCs as compared with WT VSMCs (Fig. 3b).

Characterization of homo-IR/IGF1R and hetero-IR/IGF1R on signaling. To determine the mechanisms for the enhancement of insulin signaling, we examined insulin and IGF1 binding in SMIRKO and SMIGF1RKO VSMCs. Compared with VSMCs from $W T$ mice, insulin binding was decreased by $>80 \%$ in SMIRKO VSMCs (Fig. 4a). In contrast, ${ }^{125}$ I-insulin binding was increased by twofold in SMIGF1RKO VSMCs compared with WT VSMCs (Fig. 4a). ${ }^{125}$ I-IGF1 binding was decreased by $50 \%$ in SMIGF1RKO VSMCs and by $20 \%$ in SMIRKO VSMCs compared with WT VSMC (Fig. 4b). To investigate whether the increased insulin binding to SMIGF1RKO VSMCs was due to increases of IR on the cell surface, cell surface proteins were labeled with biotin which were precipitated with avidin-conjugated beads and analyzed by immunoblotting. IR protein expressions on cell surface or in the whole cell were not different between $W T$ and SMIGF1RKO VSMCs (Fig. 4c, d). Protein levels of IGF1R were decreased on the cell surface of SMIGF1RKO VSMCs compared with WT VSMCs by $63 \%$, and not changed in SMIRKO vs. WT VSMCs (Fig. 4c, d).

Structurally, IR and IGF1R are composed of heterodimers of $a$ and $\beta$ chains conjugated by disulfide bonds ${ }^{16}$. In addition, IR and IGF1R can form hetero-IR/IGF1R receptors due to their structure similarities ${ }^{30}$. To estimate the relative levels of homo-IR, heteroIR/IGF, and homo-IGF1R receptors, cell lysates were immunoprecipitated with anti-IGF1R antibody, and the levels of IR and IGF1R in immunoprecipitation, supernatant, and cell lysate before immunoprecipitation were determined. The IR in immunoprecipitation represented IR from hetero-IR/IGF1R, whereas IR remained in supernatant were likely homo-IR/IR. IGF1R was undectable in supernatant in all three groups of cells, indicating that the difference of IR in supernatant of $W T$ and SMIGF1RKO was not due to incomplete precipitation. The results showed that $\sim 20 \%$ of IR was homo-IR receptors in WT VSMCs compared with $40 \%$ in SMIGF1RKO VSMCs, which were due to a $70 \%$ reduction of IGF1R expression (Fig. 4e, f).

To compare clearly homo-IR and homo-IGF1R signaling and activation without the presence of IR/IGF1R heterodimers, VSMCs from IR flox/flox mice were infected with AdCre to knockdown IR. IR was deficient in AdCre transfected VSMCs. Insulin-induced Akt phosphorylation was increased by 6.4 -fold in $W T$ VSMCs, but only threefold in IR-deficient VSMCs (Supplementary Fig. 10A). IGF1R flox/flox VSMCs were infected with AdCre to completely delete IGF1R. Infection with AdCredeleted IGF1R in IGF1R flox/flox VSMCs by $>99 \%$ (Supplementary Fig. 10B). Consistent with the previous findings from VSMC of SMIGF1RKO mice, insulin (100 nM)-induced p-Akt was enhanced significantly by $340 \%$, whereas IGF-1-induced p-Akt was decreased by $63 \%$ in VSMCs with IGF1R knockdown compared with WT VSMCs (Supplementary Fig. 10B). These results clearly demonstrate that homo-IR can transmit insulin signaling greater than hetero-IR/IGFIR by $340 \%$ and homoIGF1R.

To clarify further the mechanism for the enhancement of homo-IR signaling compared with hetero- IR/IGF1R or homoIGF1R, we characterized whether the increased activities were located in the IR and IGF1Ra subunit containing the ligandbinding motif or their $\beta$ subunit, which has the tyrosine kinase ${ }^{16}$. Ligand binding to the $\alpha$ subunit leads to $\beta$ subunits transautophosphorylation ${ }^{16}$. There are two possibilities for the decrease in the activation of hetero-IR/IGF1R compared with the homo-IR by insulin. The first possibility is that insulin bound less to IR $\alpha /$ IGF1a than IR $\alpha / I R \alpha$. The second possibility is that insulin has similar binding affinity to homo-IR and hetero-IR $\alpha /$ IGF1Ra, but insulin binds to IR/IGF1R is not as effective to activate IR $\beta /$ IGF1R $\beta$. To differentiate these possibilities, we studied the effects of insulin and IGF-1 to activate specifically constructed chimeric receptors which are composed of IRextracellular domain (ECD)/IGF1R-intracellular domain (ICD) or IGF1R-extracellular domain (ECD)/IR-intracellular domain (ICD). First, both IR and IGF1R were deleted completely by infecting VSMCs containing both IR flox/flox and IGF1R flox/flox with AdCre. In those double-knockout (DKO) VSMCs, with both 
a

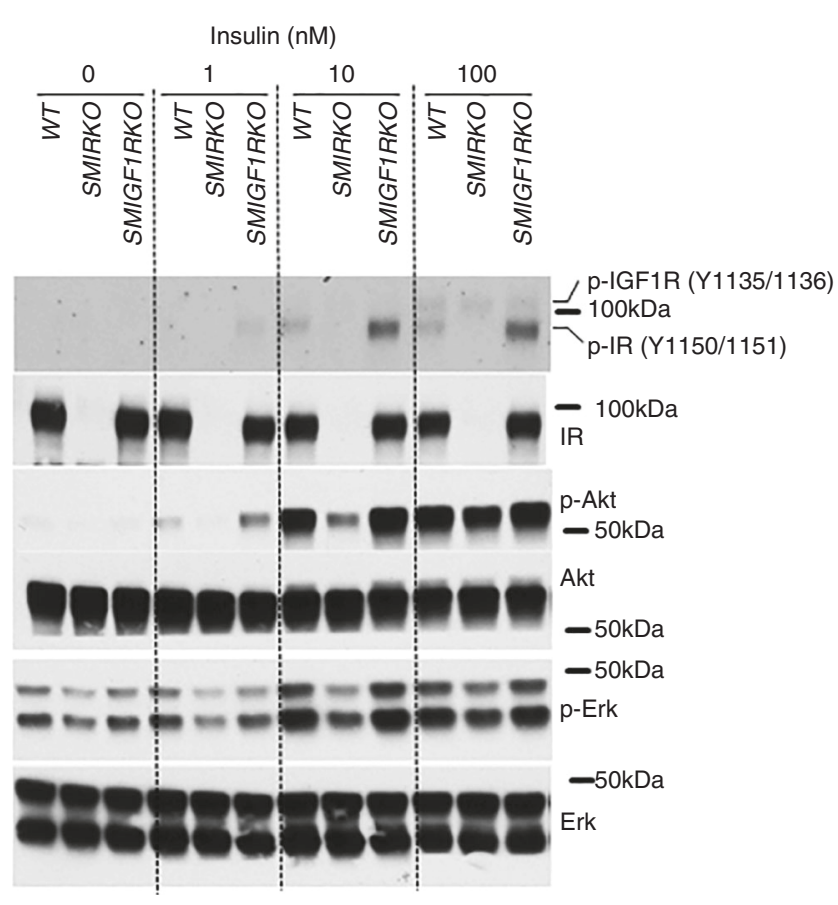

b

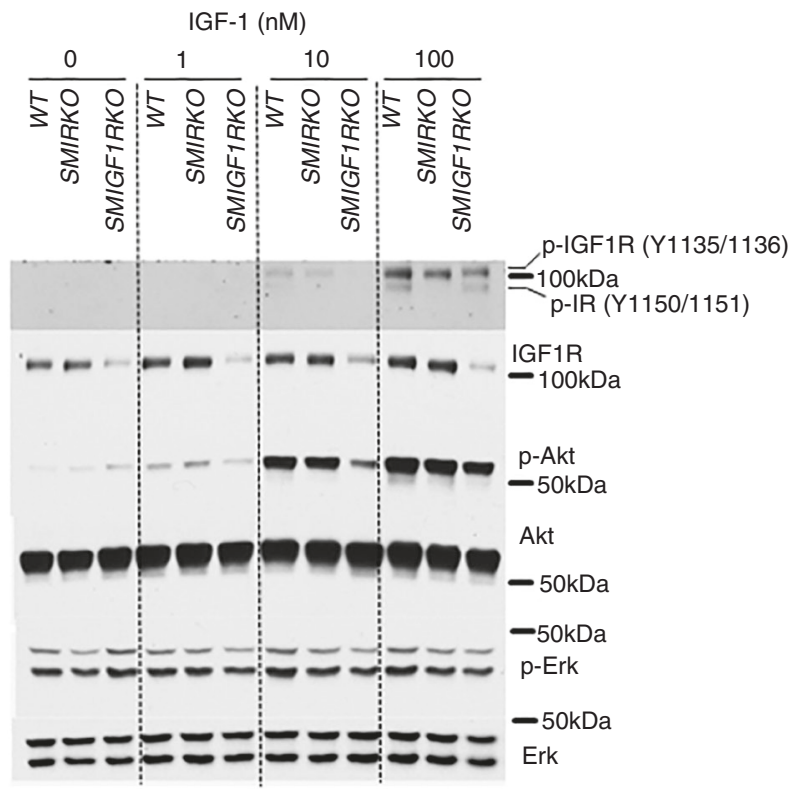

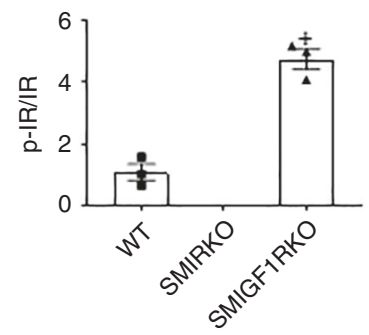
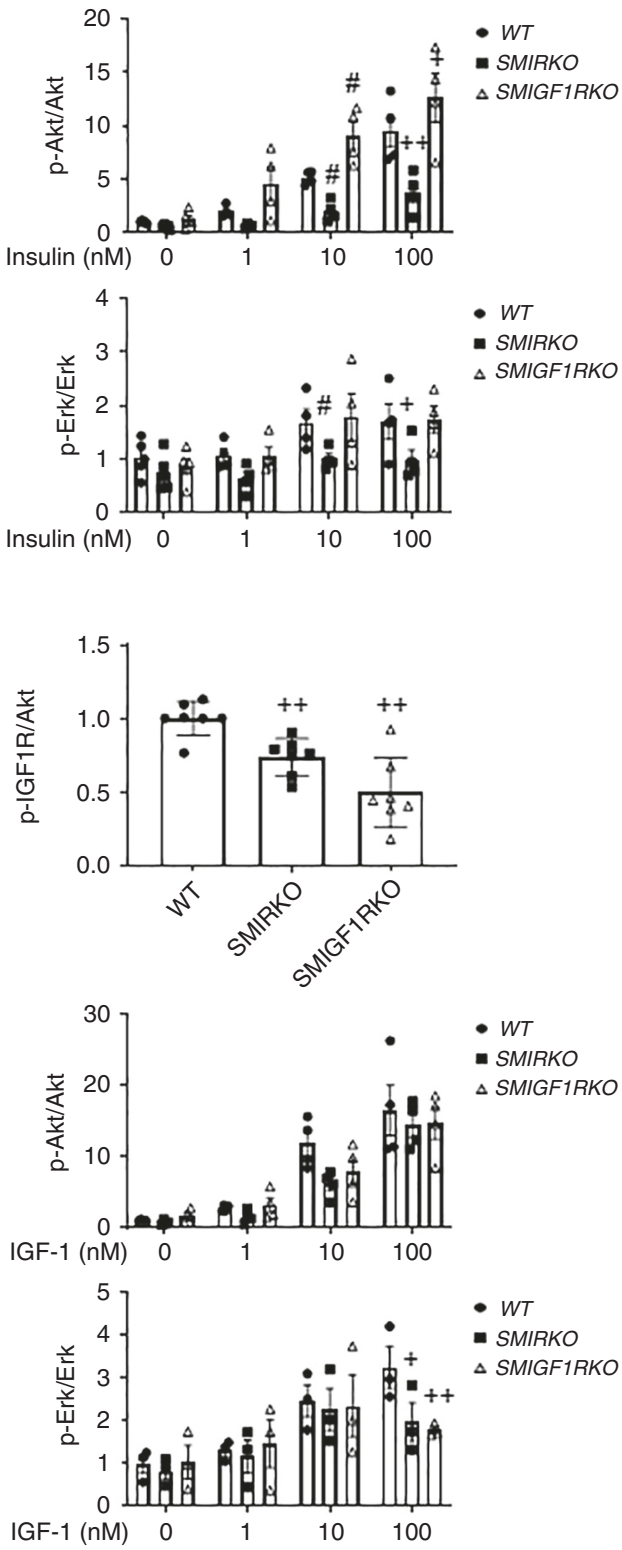

Fig. 3 Insulin and IGF-1 signaling in WT, SMIRKO, or SMIGFIRKO VSMCs. a Starved VSMCs were stimulated with different dose of insulin for 15 min (For $\mathrm{pIR} / \mathrm{IR}, n=3$ for each group; for pAkt/Akt and $\mathrm{pErk} /$ Erk, $n=5$ for each group at $0 \mathrm{nM} ; n=4$ for each group at 1, 10, and 100 nM. ${ }^{\#} p<0.05$, vs. WT stimulated with $10 \mathrm{nM}$ inslulin; ${ }^{+} p<0.05,++p<0.01$ vs. WT stimulated with $100 \mathrm{nM}$ insulin). b Starved VSMCs were stimulated with different dose of IGF-1 for $15 \mathrm{~min}$ (For pIR/IR, $n=7$ for each group; for $p A k t / A k t, n=4$ for each group and for $p$ Erk/Erk, $n=3$ for each group, $+p<0.05,++p<0.01$ vs. WT stimulated with $100 \mathrm{nM}$ (GF-1). The data are mean \pm SEM. Two-tailed $t$ test or two-way ANOVA with a post hoc test. Source data are provided as a Source Data file

IR and IGF1R deleted, either chimeric receptor IR-ECD/IGF1RICD or IGF1R-ECD/IR-ICD was overexpressed using adenovirus vectors to mediate the infection. Both endogenous IGF1R $\beta$ and IR $\beta$ expressions were decreased by $>90 \%$ in VSMC with double knockdown of IR and IGF1R (Fig. 5a, b). In VSMCs overexpressed with IR-ECD/IGF1R-ICD, the amount of the IGF1R $\beta$ was increased by 1.7 -fold compared with endogenous IGF1R $\beta$ in WT VSMCs. IR $\beta$ was increased by 13.7 -fold in VSMCs overexpressing the chimeric IGF1R-ECD/IR-ICD compared with endogenous IR $\beta$ in WT VSMCs. Insulin-induced p-Akt levels were greater in IR-extracellular domain/IGF1R-intracellular domain VSMCs by $250 \%$ than IGF1R-extracellular domain/ 
a

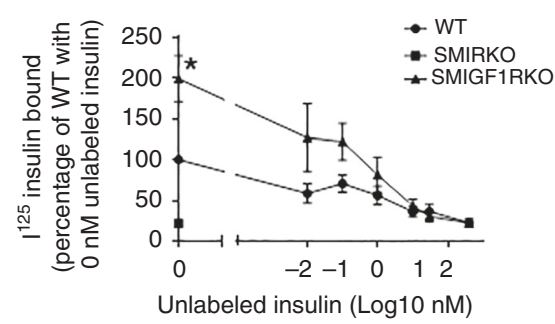

C

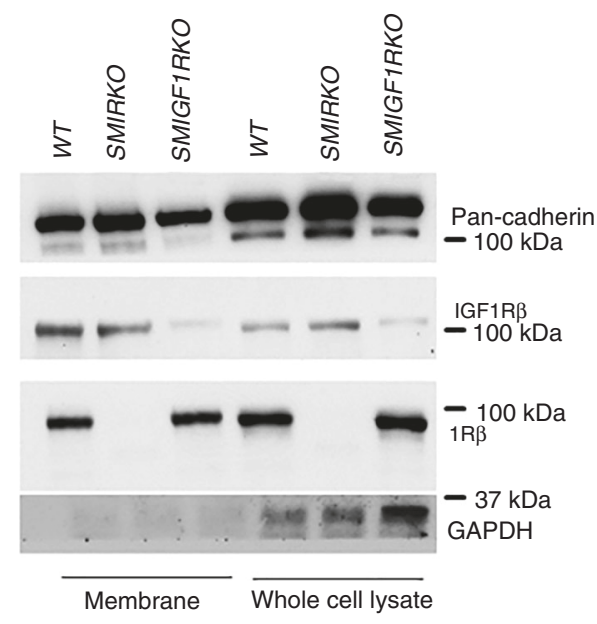

e

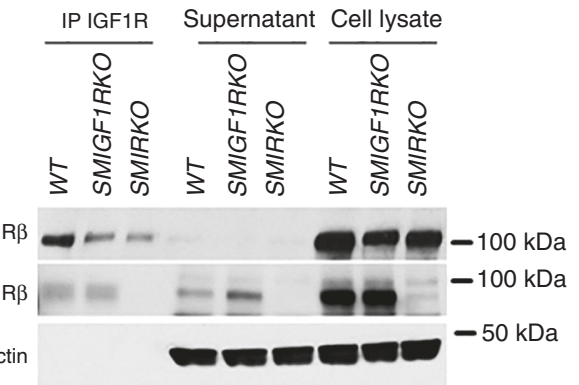

b

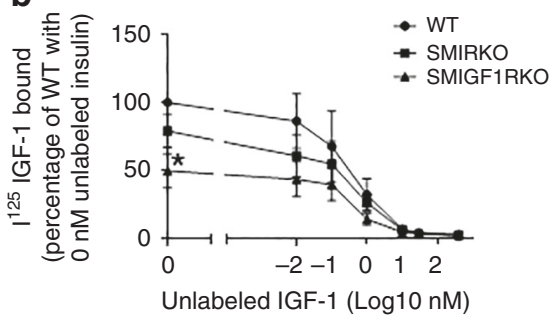

d
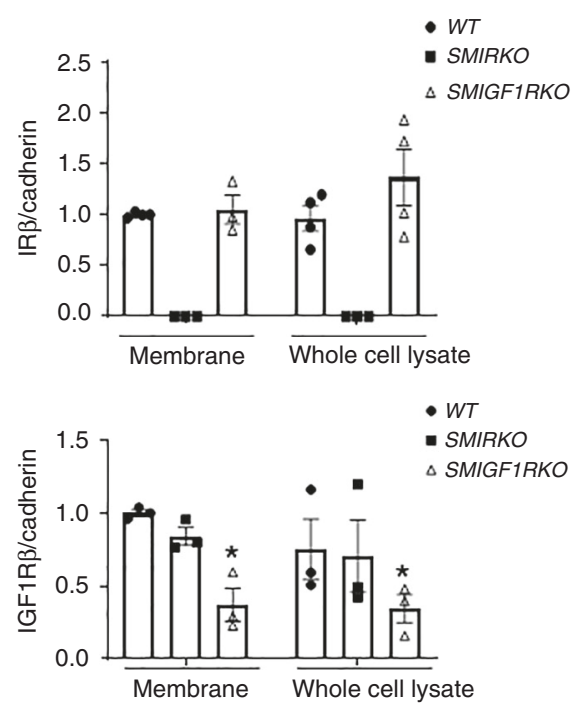

f

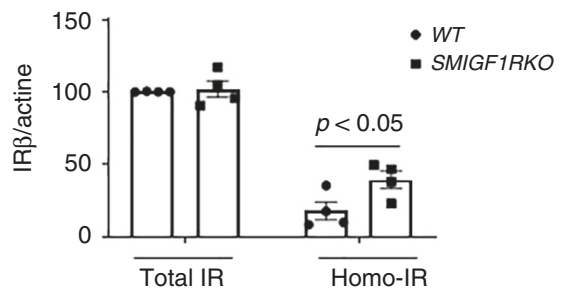

Fig. 4 Characterization of homo-IR/IGF1R and hetero-IR/IGF1R on signaling in WT, SMIRKO, or SMIGF1RKO VSMCs. a, b Insulin (a) or IGF-1 (b) binding. The VSMCs were incubated with mixture of I $^{125}$-labeled insulin and different dose of unlabeled insulin (a) or mixture of I25-labeled IGF-1 and different dose of unlabeled IGF-1 (b) for $5 \mathrm{~h}$ on ice. Insulin or IGF-1 binding was determined by scintillation counter (a, $n=5$ for WT and SMIGFIRKO at 0 nM unlabeled insulin; $n=3$ for WT and SMIGFIRKO at 0.01, 30, and $400 \mathrm{nM}$ unlabeled insulin; $n=4$ for WT and SMIGFIRKO at $0.1,1$, and $10 \mathrm{nM}$ unlabeled insulin; $\mathbf{b}, n=3$ for each group; ${ }^{*} p<0.05$ SMIGF1RKO vs. WT). c, d IR and IGF1R on cell surface. The cell membrane protein was labeled with biotin. The biotin-conjugated protein was precepitated by avidin-conjugated beads. The levels of IGF1R and IR on cell surface were determined by western blotting $(n=4$ for WT memtrane, WT total, and SMIGFIRKO total; $n=3$ for SMIRKO membrane, SMIRKO total, and SMIGF1RKO membrane, ${ }^{\star} p<0.05$ SMIGF1RKO vs. WT). e, f Ratio of homo-IR and hetero-IR/IGF1R. The cell lysates were immunoprecipitated with anti-IGF1R antibody. The levels of IR and IGF1R in immunoprecipitation, supernatant after immunoprecipitation with anti-IGF1R antibody and whole-cell lysates without immunoprecipitation were determined by western blotting. The IR in immunoprecipitation was IR from hetero-IR/IGF1R, and the remaining IR in supernatant was homo-IR. The IR in whole-cell lysates included homo-IR and IR in hetero-IR/IGF1R ( $n=4$ for each group). The data are mean \pm SEM. Two-tailed $t$ test. Source data are provided as a Source Data file

IR-intracellular domain VSMCs, whereas p-Akt induced by IGF1 in IGF1R-ECD/IR-ICD VSMCs and IR-ECD/IGF1R-ICD VSMCs were comparable (Fig. 5a-d). These results support strongly the conclusion that the enhanced effect of insulin in SMIGF1RKO vs. WT were due to enhanced binding of insulin to the IR $\alpha / \operatorname{IR} \alpha$ homodimers, and not due to activation of $\operatorname{IR} \beta / \operatorname{IR} \beta$ homodimers. To support further that IR/IR homodimers are responsible for enhancing insulin's actions in VSMCs from SMIGF1RKO mice, VSMCs with complete deletions of both endogenous IR and IGF1R were overexpressed with IR-ECD/ IGF1R-ICD alone, IGF1R-ECD/IR-ICD alone or both IR-ECD/
IGF1R-ICD and IGF1R-ECD/IR-ICD. The results showed that insulin's activation of p-Akt in cells overexpressing IRextracellular domain/IGF1R-intracellular domain was sevenfold greater than WT cells and IGF1R-extracellular domain/IRintracellular domain cells as before. In addition, insulin's effect on p-Akt in cells expressing IR-ECD/IGF1R-ICD and IGF1RECD/IR-ICD together were greatly decreased compared with cells which expressed only IR-ECD/IGF1R-ICD (Fig. 5e, f).

Insulin targets in VSMCs. To identify the specific downstream cellular targets of IR that are mediating its enhanced effects on 
a
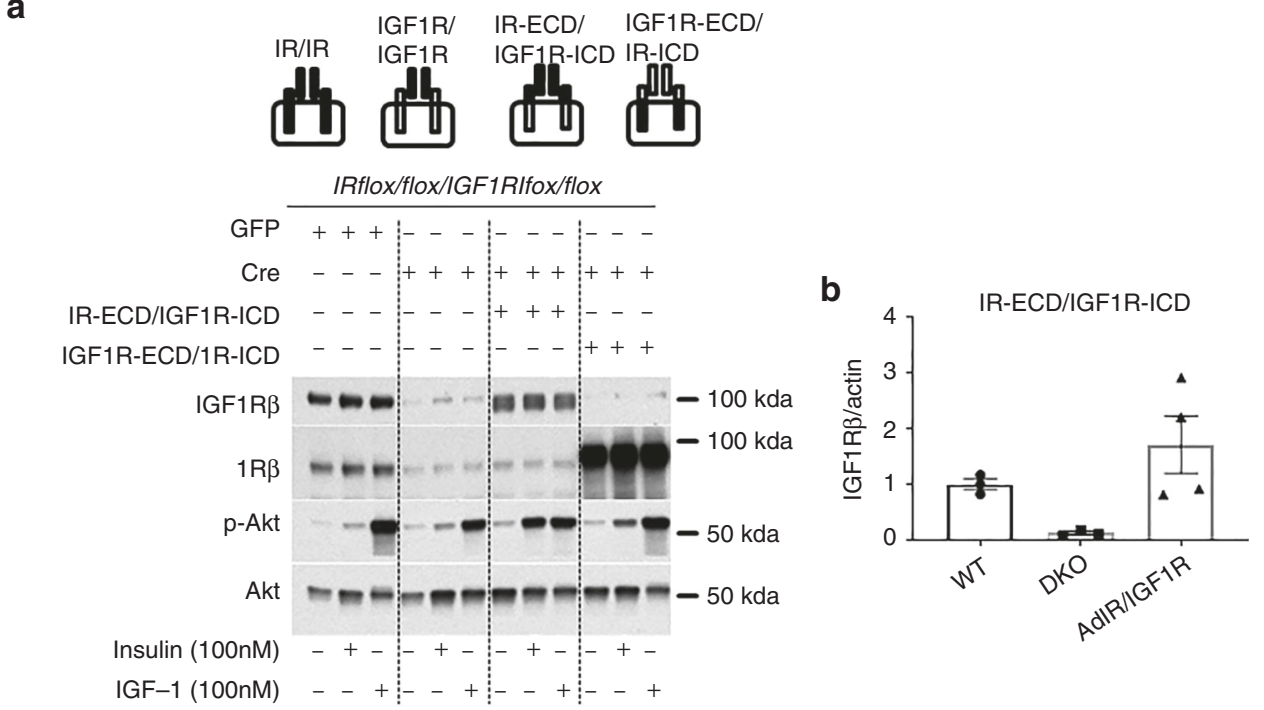

C

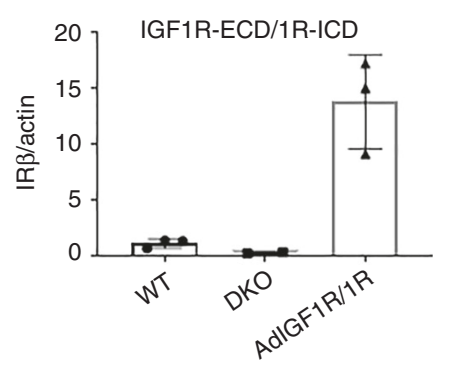

e

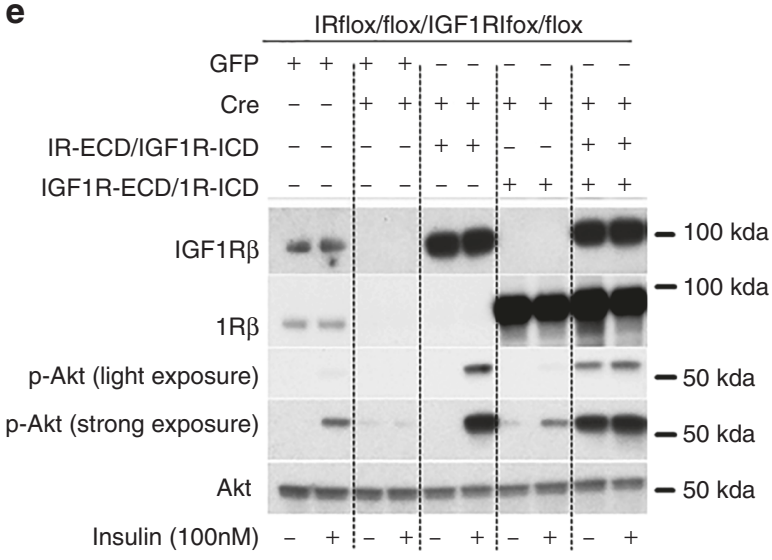

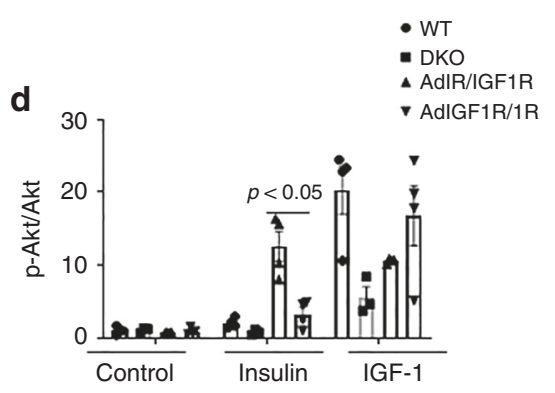

f

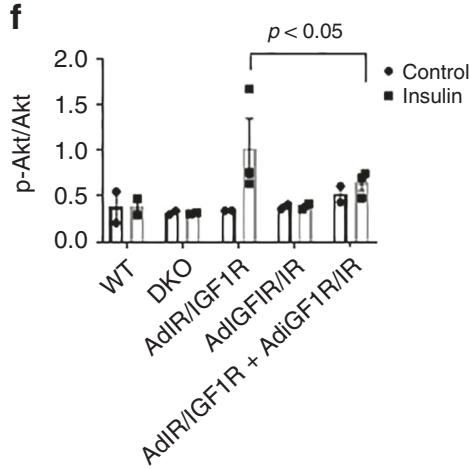

Fig. 5 Insulin and IGF-1 action on chimeric IR/IGF1R receptors. VSMCs were cultured from aortas of mice which had both IRflox/flox and IGFIRflox/flox

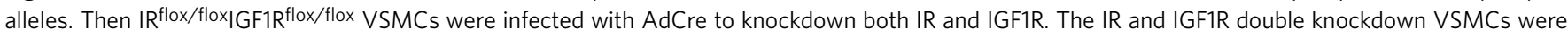
further infected with AdIR-ECD/IGF1R-ICD or AdIGF1R-ECD/IR-ICD or both to overexpress chimeric IR-ECD/IGF1R-ICD or IGF1R-ECD/IR-ICD receptors. a, e indicate representative western blotting images. b-d, $\mathbf{f}$ show summarized quantification data $(\mathbf{b}, n=3$ for WT and DKO, $n=4$ for AdIR/IGF1R; $\mathbf{c}, n=3$ for each group; $\mathbf{d}, n=4$ for WT, AdIR/IGF1R, and AdIGF1R/IR, $n=3$ for DKO; $\mathbf{f}, n=3$ for each group). The data are mean \pm SEM. Two-way ANOVA with a post hoc test. Source data are provided as a Source Data file

VSMC proliferation and intimal hyperplasia, cultured VSMCs with IGF1R deletion of $>99 \%$ derived by infecting VSMCs from SMIGFIRKO mice with AdCre and VSMC from WT mice were stimulated with insulin at $100 \mathrm{nM}$, and changes in gene expression were evaluated by RNA-seq analysis (data shown in Supplementary Table 1). Insulin increased the expression of 13 genes by $>1.5$-fold in IGF1R KO VSMC than WT VSMC. These genes were evaluated further in the media of the aorta from $W T$, SMIRKO, and SM1GF1KO as shown in Fig. 6. Of these 13 genes, only hyaluronan synthesis 2 (Has2) was decreased in the aorta from SMIRKO mice compared with WT mice by $>50 \%(p<0.05)$ (Fig. 6a, b). Furthermore, Has 2 mRNA and protein expressions were increased in the aorta of SMIGF1RKO mice compared with WT mice (Fig. 6c). These results suggest that changes in Has 2 could be a specific cellular target of IR in VSMC to enhance DNA synthesis. To evaluate the regulation of Has 2 expression by insulin, cultured VSMC from WT and SMIGF1R KO mice were compared. Insulin $(10 \mathrm{nM})$ significantly increased Has 2 mRNA 

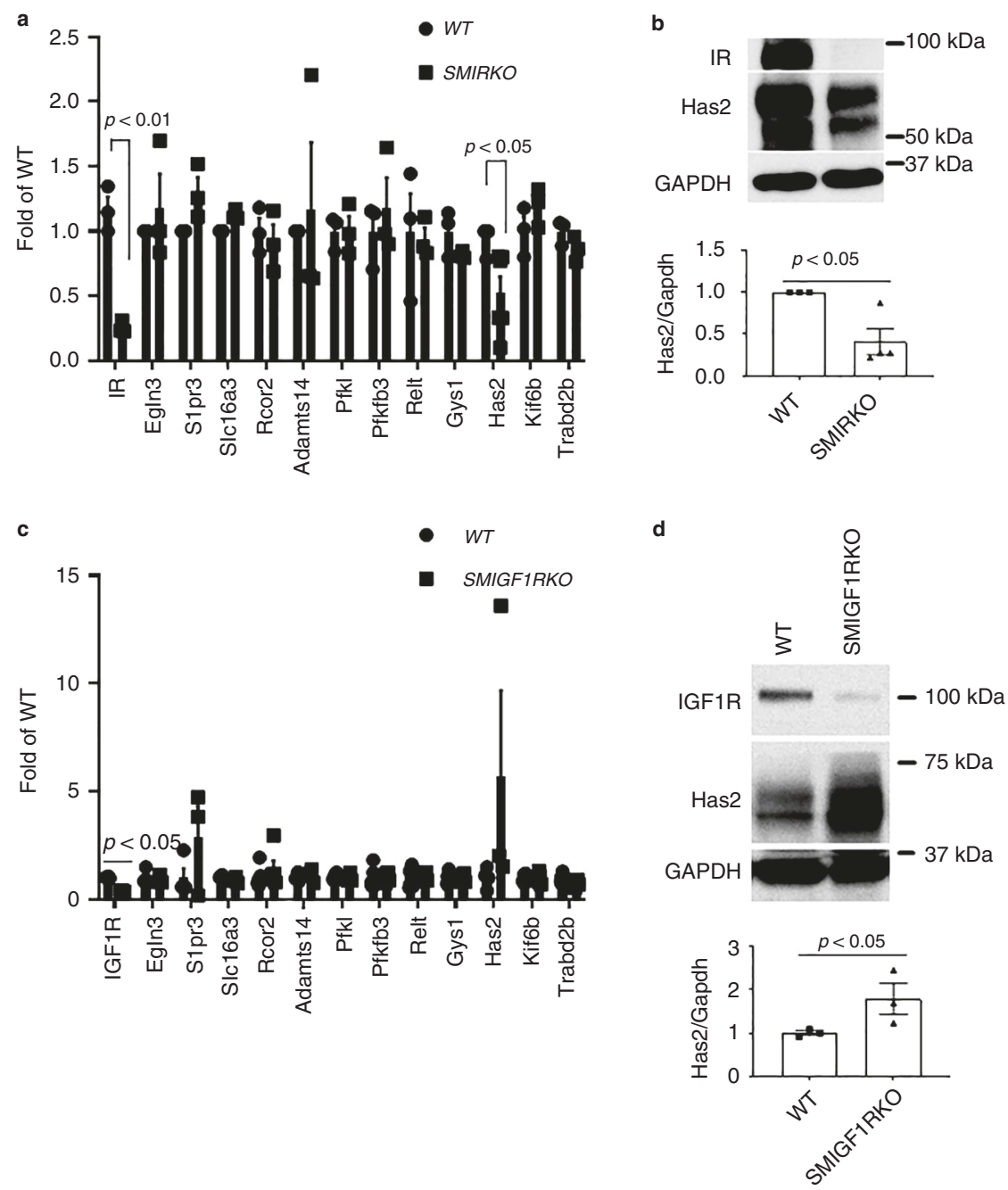

Fig. 6 Insulin targets in VSMCs. a The expression of insulin-targeted genes in aorta media of WT and SMIRKO mice. RNA was extracted from aorta media of $W T$ and SMIRKO mice and the expression levels of genes were determined by qPCR (WT Has2, $n=5 ;$ SMIRKO Has $2, n=6 ; n=3$ for each of the remaining groups). $\mathbf{b}$ The expression of Has 2 protein in aorta media of WT and SMIRKO mice $(W T, n=3 ; S M I R K O, n=4)$. c The expression of insulintargeted genes in aorta media of WT and SMIGFIRKO mice. RNA was extracted from aorta media of WT and SMIGF1RKO mice, and the expression levels of genes were determined by qPCR ( $n=3$ per group). d The expression of Has 2 protein in aorta media of WT and SMIGFTRKO mice ( $n=3$ per group). The data are mean \pm SEM. Two-tailed $t$ test. Source data are provided as a Source Data file

levels at $1 \mathrm{~h}$ by $248 \%$ in SMIGF1RKO VSMCs (Fig. $7 \mathrm{a}, \mathrm{b}$ ). In addition, insulin also increased hyaluronan content in the culture media of WT VSMC, whereas SMIRKO VSMC did not respond to insulin. In contrast, insulin significantly increased hyaluronan content in the medium of SMIGFIRKO VSMC (Fig. 7c).

The expression of Has 2 gene was further investigated in vivo using femoral artery 9 days after wire injury in WT (IR flox/flox), SMIRKO, WT (IGF1R flox/flox), and SMIGF1RKO mice on HFD diet (Fig. 7d). The expression of Has 2 mRNA was decreased in femoral artery of SMIRKO mice compared with its WT control (IR flox/flox mice). In contrast, the mRNA expression of Has2 in the femoral artery was significantly increased in SMIGFIRKO mice vs. its WT control, IGF1R flox/flox mice. The intensity of hyaluronan staining as levels in the intimal area of femoral artery of SMIGF1RKO mice were significantly increased compared with $W T$ (IGF1R flox/flox), as well as to IR flox/flox and SMIRKO mice (Fig. 7e, f).

Analysis of insulin signaling pathways, which could be responsible for regulating Has2 expression, was also studied using VSMC from SMIGF1RKO and WT mice. Again, insulin increased Has2 mRNA levels greater in SMIGF1RKO VSMC than
WT VSMC (Supplementary Fig. 11). Small-molecule inhibitors of PI3 Kinase (wortmannin) and MEK (PD98059) inhibited completely the expression of insulin-induced Has2 expression (Supplementary Fig. 11).

FoxO and mTOR are the two major downstream targets of Akt. Insulin increased phosphorylation of FoxO1 at threonine 24 and FoxO3a at threonine 32 (Supplementary Fig. 12). Recently, it was reported that FoxO1 could bind to the promoter of Has2 and inhibit the transcription of $\operatorname{Has}^{31}$. Thus, we assessed the effect of insulin to regulate Has2 gene expression by infecting VSMCs with adenovirus containing constitutively active FoxO1 (CA-FoxO1) with mutating threonine 24 to alanine, serine 253 to aspartic acid, and serine 356 to alanine. CA-FoxO1 cannot be phosphorylated by insulin or other growth factors. VSMCs were infected with adenovirus containing either GFP or CA-FoxO1, and the expression of Has2 with or without insulin stimulation was determined by qPCR. The basal level of Has 2 was increased significantly in VSMCs overexpressed with CA-FoxO1. Insulin's effect to induce Has2 gene expression was inhibited in VSMCs infected with CA-FoxO1 (Fig. 7g). Since CA-FoxO1 can increase Akt phosphorylation to activate $\mathrm{mTORC1}^{32}$, the increased basal 


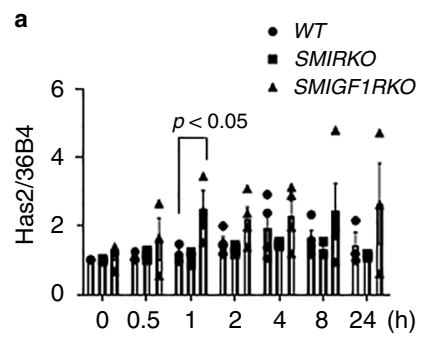

b
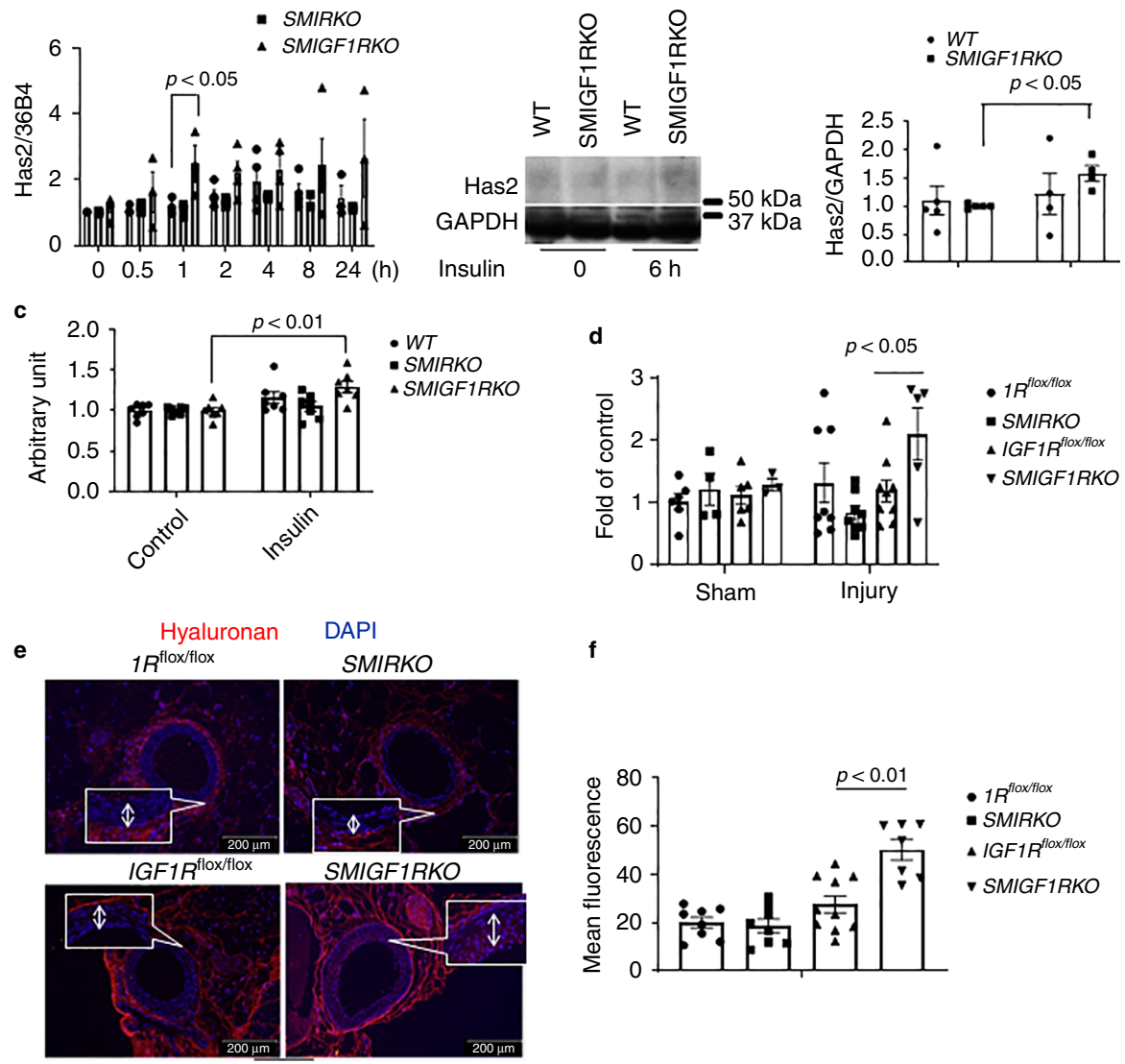

$c$

f

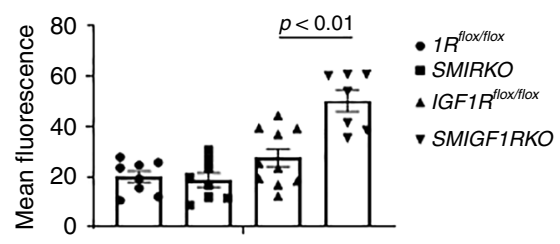

g
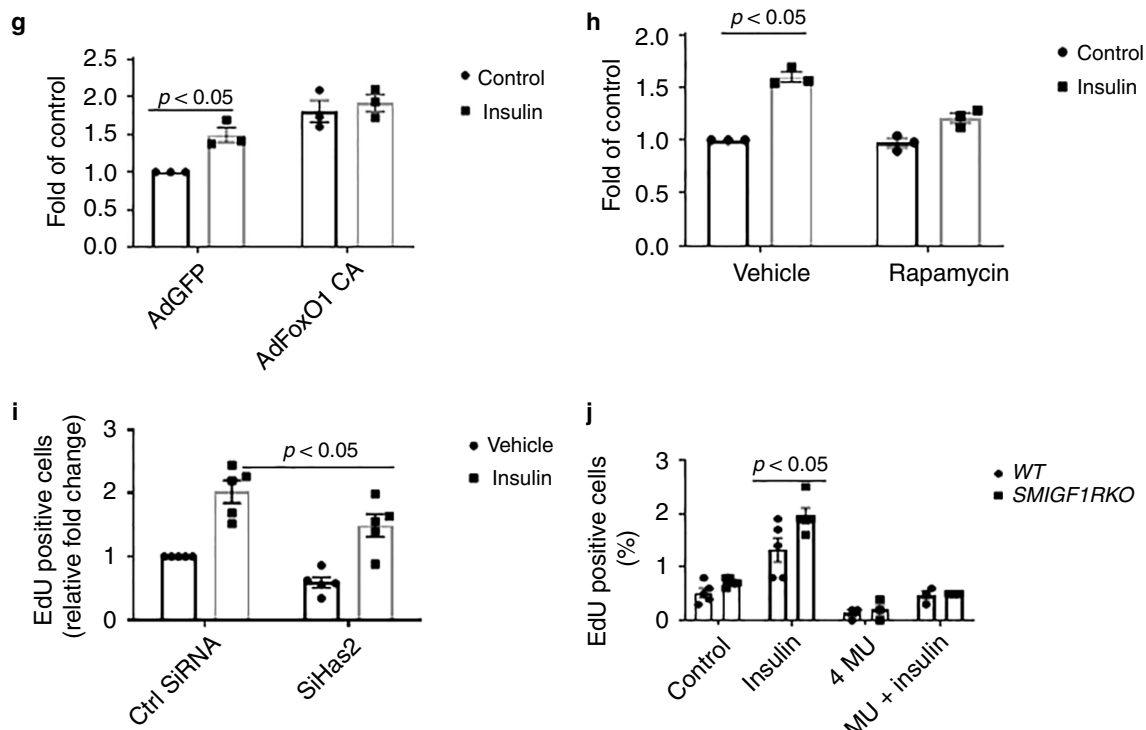

j

- Vehicle

- Insulin

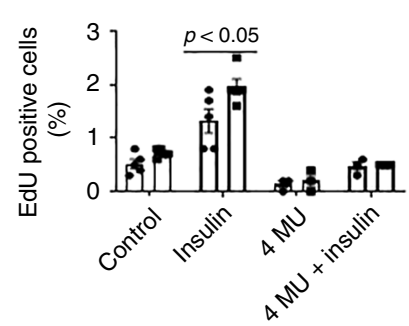

WT

- SMIGF1RKO

Fig. 7 Has2 expression in VSMCs and femoral artery. a Starved WT, SMIRKO, or SMIGF1RKO VSMCs were stimulated with $10 \mathrm{nM}$ insulin, and the gene expression of Has 2 was determined by qPCR ( $0 \mathrm{~h}, n=4$ for each group; $0.5 \mathrm{~h}, n=3$ for each group; $1 \mathrm{~h}, n=3$ for each group; $2 \mathrm{~h}, n=4$ for each group; $4 \mathrm{~h}$ and $8 \mathrm{~h}, n=4$ for WT and SMIGFIRKO, $n=3$ for SMIRKO; $24 \mathrm{~h}, n=3$ for WT and SMIGFIRKO; $n=2$ for SMIRKO). b Has2 protein expression in VSMCs treated with insulin. c Hyaluronan in cultured medium. The hyaluronan content before and $12 \mathrm{~h}$ after $10 \mathrm{nM}$ insulin stimulation was determined by ELISA. d The femoral arteries were

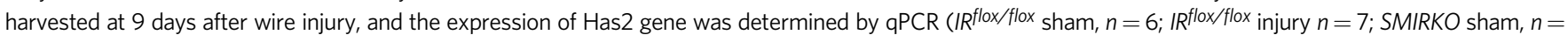
4; SMIRKO injury, $n=8$; IGFIR flox/flox sham, $n=6$; IGFIRflox/flox injury, $n=9$; SMIGFIRKO sham, $n=3$; SMIGFIRKO injury, $n=5$ ). e, $\mathbf{f}$ Hyaluronan staining in wireinjured femoral artery. Hyaluronan staining was performed on feoral arteries at 4 weeks after wire injury (arrow indicates neointima. IRflox/flox,$n=8 ; S M I R K O, n=$ 8; IGFIR flox/flox,$n=9$; SMIGFIRKO, $n=7$ ). $\mathbf{g}$ VSMCs were infected with AdFoxO1 CA or AdGFP and then stimulated with insulin for $4 \mathrm{~h}$. The expression of Has2 gene was determined by qPCR ( $n=3$ per group). $\mathbf{h}$ VSMCs were treated with rapamycin $(10 \mathrm{ng} / \mathrm{ml})$ for $1 \mathrm{~h}$ and then stimulated with insulin for $4 \mathrm{~h}$. Expression of Has2 gene was determined by qPCR ( $n=3$ per group). i The expression of Has2 in VSMCs was knockdown by siRNA, and cellular proliferation was determined by EdU incorporation ( $n=8$ for each group). $\mathbf{j}$ Starved VSMCs were pretreated with $1 \mathrm{mM} 4-\mathrm{MU}$ for $4 \mathrm{~h}$ and then stimulated with $100 \mathrm{nM}$ insulin for $24 \mathrm{~h}$. The cell proliferation was determined by measuring EdU-positive cells by flow cytometry ( $n=3$ per group). The data are mean \pm SEM. Two-tailed $t$ test or two-way ANOVA with a post hoc test. Source data are provided as a Source Data file 
level of Has2 in CA-FoxO1 overexpressed cells could be due to the activation of mTORC1. Thus, we incubated VSMCs with rapamycin, which is a mTORC1 inhibitor. The presence of rapamycin partially inhibited insulin-induced Has 2 gene expression (Fig. $7 \mathrm{~h}$ ).

To demonstrate that Has2 and hyaluronan play an important role in VSMC proliferation, we reduced Has 2 expression by siRNA and then studied its reduction on cellular proliferation. siRNA decreased Has 2 gene expression by $67 \%$ compared with control (Supplementary Fig. 13). Cellular proliferation was decreased significantly by $39 \%$ at the basal level and $31 \%$ after insulin stimulation in Has 2 knockdown cells (Fig. 7i). The effect of hyaluronan inhibitor 4-MU on VSMC proliferation was studied. As shown in Fig. 7j, insulin (100 nM) greatly elevated Edu incorporation in VSMCs from SMIGF1RKO compared with VSMCs from WT Mice. The addition of 4-MU significantly inhibited both insulin (Fig. 7j) and 10\% fetal bovine serum (Supplementary Fig. 14A) induced Edu incorporation, respectively. To determine the effects of 4-MU on cell death, VSMC were treated with $4-\mathrm{MU}$ for $24 \mathrm{~h}$, and stained cells with Annexin $\mathrm{V}$ and PI and assessed by flow cytometry. Treatment with 4-MU increased dead cells (including apoptosis and necrosis) from 18 to 44\% (Supplementary Fig. 14B).

\section{Discussion}

Prevailing theory has been that the elevations of circulating IGF-1 and insulin levels in insulin resistance and type 2 diabetes are both partly responsible for the acceleration of VSMC proliferation and restenosis causing stent failure in the treatment for coronary artery disease ${ }^{15,33}$. The major reason for the difficulty in identifying the individual contributions of insulin and IGF1 is the almost complete overlap of cellular signaling and actions of these hormones and their receptors in the VSMC ${ }^{6,34}$. Our findings using specific deletions of IR and IGF1R in VSMC provide the surprising and clear result that the loss of IGF1R does not protect against intimal hyperplasia, whereas deletion of IR decreases VSMC proliferation and intimal hyperplasia following intimal injury. These surprising results strongly suggest that insulin and IR are primarily responsible for the increased risk of restenosis in insulin resistance and diabetes. The findings that insulin and IGF1 receptors on VSMC proliferation were different is surprising, considering that almost all the biological actions of these hormones are overlapping in most cell types ${ }^{35}$. In fact, most studies have concluded that IGF-1 and IGF1R are the major contributors to VSMC growth due to their greater mitogenic actions at physiological levels than insulin on VSMC cells. ${ }^{6,10}$. However, these results suggest that the accelerated restenosis in insulin resistance is mainly due to insulin action via homo-IR receptor and may even be inhibited by IGF1R in VSMCs. The important role of insulin and IR on intimal hyperplasia has been clearly demonstrated in this study by comparing the extent of the intimal hyperplasia to injury in Myh11IRKO vs. SMIGF1KO mice on HFD. This mouse model replicated diabetes and insulin resistance in people by exhibiting elevated IGF-1 and insulin levels, insulin resistance, and enhanced intimal hyperplasia when they are fed HFD vs. control. This pathology of intimal hyperplasia also exhibited predominantly migration and proliferation of VSMC with minor contribution from inflammation, which reflects restenosis clinically ${ }^{3,4}$.

In vivo and in vitro studies of VSMC proliferation confirmed the pathological findings that showed the deletion of IR decreased mitogenic effects of insulin. Even more surprising was the finding that insulin's signaling and actions were actually increased to a greater extent in VSMC isolated from SMIGF1RKO compared with WT mice which contrasted with the decreases in IGF1 effects in SMIRKO cells. In addition, these results suggested that the loss of IGF1 receptor actually enhanced IR cellular effects. These findings indicated that structural differences in IR and IGF1R can mediate different specific actions in VSMC, which is unlike many other cell type that have been studied ${ }^{36}$.

The potential mechanisms for the selective and enhanced action of insulin on VSMC with IGF1R deletion was related to the increased binding of insulin to the homo-IR when compared with hetero-IR/IGF1R or homo-IGF1R. In addition, the presence of IGF1R in VSMC may even inhibit insulin and IR actions by the formation of hetero-IR/IGF1 receptors. This conclusion is supported by several studies, including binding experiments, actions in VSMCs containing pure homo-IR or IGF1 receptor in doubleknockout cells, and the use of chimera IR/IGF1 receptors. The increased binding of insulin to IR in SMIGF1RKO mice or VSMC with IGF1R deletion is likely due to the increase of binding affinity, since no changes in IR levels of either $\alpha$ or $\beta$ subunit of IR were observed at cell surface or at whole-cell level. These results indicated that there are significant differences in the binding properties to homo-IR versus to homo-IGF1 receptors by insulin and IGF-1 to their respective receptors. The additional results from the chimera IR-ECD/IGF1R-ICD versus IGF1R-ECD/IRICD receptors in VSMC with double knockout of IR and IGF1R further documented important differences in the binding properties of insulin to IR versus IGF1R $\alpha$-binding subunits. The differential enhancement of insulin binding to homo-IR receptors and the inhibition of insulin actions with the addition of both IRECD/IGF1R-ICD and IGF1R-ECD/IR-ICD clearly demonstrate the structural differences in the binding region of the IRa subunits versus IGF1Ra subunits especially in the cysteine-rich regions which has been previously noted to be quite different between these two receptors ${ }^{37,38}$. The p-Akt induced by Insulin was slightly higher than that by IGF-1 in IR-ECD/IGF1R-ICD cells, although the difference was not significant. The cells were stimulated with $100 \mathrm{nM}$ insulin or $100 \mathrm{nM}$ IGF1. These data suggest that the extracellular domain of insulin receptor could be fully activated by high dose of IGF-1, whereas the extracellular domain of IGF-1 receptor could only be partially activated by high dose of insulin. Furthermore, the increase of basal activation of Akt in double-chimeric SMCs (Fig. 5e) might be due to autoactivation of double-chimeric receptors by paracrine production of IGF-1. Since double-chimeric receptors formed hybrid chimeric receptor (IR-ECDIGF1R-ECD/IGF1R-ICDIR-ICD), another possibility might be the hybrid chimeric receptor was more sensitive to the trace amount of IGF-1 produced by smooth muscle cells.

The data from the chimera receptor studies also support that the interactions of IRa-binding subunits have synergistic effects in the transmission of insulin signaling from $\alpha$ to $\beta$ subunit of IR or IGF1R. Our finding in smooth muscle cells are partially different to those observed in preadipocytes using the same chimeric receptor ${ }^{36}$, which indicated that the metabolic and growth promoting actions of insulin and IGF-1 receptors are similar but they varied in actions due to differences in interaction between the $\beta$ subunits of these hormone receptors and intracellular substrates. Our data clearly demonstrate that the a-binding subunit of these receptors can also have major contributions to their cellular actions, especially regarding mitogenic effects of VSMC. The finding of IGF1R could potentially be a negative regulator of insulin action has previously been suggested by Kearney et al., who reported that the presence of IGF1R negatively impacted insulin's regulation of NO actions in the endothelium ${ }^{39}$. Our study clearly demonstrates that the insulin binding is decreased by the formation of hetero-IR/IGF1R, which decreased insulin's signaling in the VSMC resulting in the reduction of VSMC migration and proliferation after injury. 
This study was extended further by RNA-seq analysis of insulin-stimulated gene expression in VSMC, which identified Has2 as a potential downstream target of homo-IR receptors that could be mediating the enhanced insulin actions for VSMC proliferation and intimal hyperplasia. This selective action of IR to increase expression of Has 2 is supported by the findings that insulin increased Has 2 expression in VSMC and in vivo from SMIGF1RKO mice to a greater extent than in WT VSMC. Thus, we have identified a target of insulin and IR in VSMC. The function of Has2 is to produce hyaluronan, an important component of the extracellular matrix and critical for VSMC proliferation, migration, and differentiation ${ }^{40-42}$. Has 2 is the major isoform of Has in VSMC and has a critical role in cardiovascular tissue formation, since embryotic deletion of Has2 is lethal with abnormal development of the heart and large vessels ${ }^{43}$. In intimal hyperplasia models, hyaluronan production increases after balloon injury with enhanced levels in the media and even in restenosis of human arteries ${ }^{44,45}$. Our study clearly supports the importance of Has2 and its actions in VSMC proliferation. In addition, treatment with an inhibitor of Has2 decreases insulininduced proliferation of VSMC. The mechanism of insulin action to increase Has 2 expression appears to be mediated through multiple pathways, including both AKT and MAPK kinase. Other studies have shown that the direct activation of MAP kinase can regulate Has 2 gene expression ${ }^{46}$; however, our findings suggest that p-Akt may also be important.

In summary, we find that insulin through homo-IR receptor is most likely a significant cause for enhancing VSMC proliferation in restenosis. The identification of the selective actions of homoIR that can be inhibited by IGF1R is exciting especially with the identification of a IR-specific downstream target, Has2, which has already shown to be important for restenosis and possible atherosclerosis. These results suggest inhibition of homo-IR in VSMC at the stent site could be a potential clinical target for treatment in reversing the increase risk of restenosis after stents and angioplasty in people with diabetes and insulin resistance.

\begin{abstract}
Methods
Animals. $\mathrm{IR}^{\text {flox/flox }}, \mathrm{IGF} \mathrm{R}^{\text {flox/flox }}$, and $\mathrm{IR}^{\text {flox/flox}} / \mathrm{IGF} 1 \mathrm{R}^{\text {flox/flox }}$ mice were kindly provided by Dr. C. Ronald Kahn (Joslin Diabetes Center, Boston, MA, USA). SM22 $\alpha$ Cre and Myh11-Cre mice were obtained from Jackson Laboratory. All of mice have been backcrossed with C56BL/6J for more than six times. Male mice were fed a high-fat diet (Research diet, $60 \%$ calories from fat, D12492) starting at 4 weeks of age and continuing for 8 weeks. All protocols for animal use and euthanasia were reviewed and approved by the Animal Care Committee of the Joslin Diabetes Center. They are in accordance with NIH guidelines following the standards established by the Animal Welfare Acts and by the documents entitled "Principles for Use of Animals" and "Guide for the Care and Use of Laboratory Animals".
\end{abstract}

Femoral artery wire injury and artery remodeling analysis. Bilateral wire injury of the femoral artery was performed ${ }^{14}$. In brief, the femoral arteries were injured by three passages of a 0.014 -inch-diameter angioplasty guidewire. The femoral arteries were harvested after perfusion with $10 \%$ formaldehyde under $75 \mathrm{mmHg}$ pressure. Paraffin-embedded 5- $\mu \mathrm{m}$ sections were stained with the Elastin staining kit (Sigma). Sections were masked for the measurement of the luminal, intimal, and medial areas using NIH ImageJ.

Smooth muscle cell culture. Mice aortas were dissected and digested with Dulbecco's modified Eagle medium (DMEM) containing $0.1 \%$ collagenase II (Worthington Biochemical Corp, Lakewood, NJ, USA) for $20 \mathrm{~min}$. The adventitia of aorta was stripped off, and the media of aorta was cut into small pieces. Then the aorta pieces were digested with the DMEM containing $0.2 \%(\mathrm{w} / \mathrm{v})$ collagenase type 1 (Worthington Biochemical Corporation, Lakewood, NJ, USA) and $0.1 \%$ BSA at $37^{\circ} \mathrm{C}$ for $1 \mathrm{~h}$. After washing and centrifuge, the cells were plated on dishes coated with $0.1 \%(w / v)$ collagen I (BioCoat, BD BioSciences, Franklin Lakes, NJ, USA) and were grown in the DMEM with $20 \%(\mathrm{v} / \mathrm{v})$ fetal bovine serum. The cells were used to do experiments at passages of $2-4$.

Lung endothelial cells culture. The lung was cut into small pieces and digested with $0.2 \%$ collagenase I 1 (Worthington Biochemical Corporation, Lakewood, NJ) with $0.1 \%$ BSA I for $1 \mathrm{~h}$. The digest was pipetted and filtered through a $100-\mu \mathrm{m}$ strainer. After centrifuge, the cells were plated into dish coated with $0.2 \%$ gelatin The endothelial cells were purified with anti-ICAM2 antibody (BD Bioscience, San Jose, CA, USA)-conjugated magnet beads twice when the cells were confluent. Endothelial cells were used to do experiments at the passages of 3-4.

Isolate adipocytes. Adipocytes were isolated from epididymal fat. The epididymal fat was cut into small pieces and digested with $10 \%$ collagenase II in DMEM/0.1\% BSA for $20 \mathrm{~min}$. The digest was centrifuged at $300 \mathrm{~g}$ for $3 \mathrm{~min}$. The adipocytes floating on the top of liquid were collected for western analysis.

Generate MyH11-CreERT2/IRfox/flox mice. MyH11-CreERT2 mice were purchased from Jackson Lab (Bar Harbor, ME, USA). IRflox/flox mice were cross-bred with MyH11-CreERT2 mice to generate MyH11-CreERT2/IRfox/flox (MyH11IRKO) mice. Insulin receptor in smooth muscle cells in MyH11IRKO mice was deleted by tamoxifen intraperitoneal injection (Sigma, St. Louis, MO, USA, $50 \mathrm{mg} / \mathrm{Kg}$ body weight) once every $24 \mathrm{~h}$ for a total of 10 consecutive days. The control mice were IRflox/flox mice which were also received tamoxifen intraperitoneal injection ( $50 \mathrm{mg} / \mathrm{Kg}$ body weight) once every $24 \mathrm{~h}$ for a total of 10 consecutive days.

Western blotting. Mouse aorta smooth muscle cells were incubated with DMEM containing $0.1 \%$ BSA for $48 \mathrm{~h}$ and then were stimulated with insulin (Sigma, St. Louis, MO, USA) or IGF1 (Gold Biotechnology Inc., St. Louis, MO, USA) for $15 \mathrm{~min}$. To observe the actions of insulin in vivo, insulin $(10 \mathrm{mIU} / \mathrm{g}$ body weight was administered to mice via intravenous injection, and aortas were harvested at 5 min after insulin injection. Tissues were placed in a heavy duty pouch and pulverized on dry ice. The cells or tissues were lysed with RIPA buffer (buffer $(50 \mathrm{mM}$ Tris- $\mathrm{HCl}, 150 \mathrm{mM} \mathrm{NaCl}, 1 \% \mathrm{NP}-40,0.5 \%$ sodium deoxycholate, and $0.1 \% \mathrm{SDS}$ ). The protein concentration was determined by BCA protein assay reagent (Thermo Scientific, Rockford, IL, USA). Protein samples were separated by electrophoresis in a $7.5 \%$ Tris- $\mathrm{HCl}$ polyacrylamide gel and transferred to a PVDF membrane, which was blocked with $5 \%$ nonfat dry milk in Tris-buffered saline- $0.1 \%$ Tween-20 and incubated with primary antibody in $4{ }^{\circ} \mathrm{C}$ overnight. Detection was carried out using an ECL Plus Western Blotting Detection kit (Thermo Fisher Scientific, Rockford, IL, USA). The membranes were incubated with Restore Plus Western Blot Stripping buffer (Thermo Fisher Scientific, Rockford, IL, USA) for 15 min to remove primary and secondary antibodies. After washing for six times with PBS $/ 0.1 \%$ Twen-20, the membranes were blotted with new antibodies. Quantitative densitometry was performed using ImageJ. Antibodies are listed in Table 1.

Glucose and insulin tolerance tests. Mice were fasted overnight and then glucose ( $2 \mathrm{mg} / \mathrm{g}$ body weight) or fasted for $6 \mathrm{~h}$, and then insulin $(0.75 \mathrm{mIU} / \mathrm{g}$ body weight $)$ was administered to mice via intraperitoneal injection. Blood was sampled from tail vein for glucose determination at $0,30,60,120 \mathrm{~min}$ after glucose or insulin injection. Glucose was measured by Contour glucometer (Bayer, Mishawaka, IN, USA).

Blood pressure assessment. Blood pressure was assessed with the Visitech noninvasive blood pressure analysis system. Up to six mice in each strain group were monitored for $5 \mathrm{~min}$ over the course of 5 days. Conscious mice were placed on a heated pad, and a tail cuff was placed in order to get blood pressure measurements.

Immunofluorescence. The femoral arteries of mice were subjected to wire injury After surgery, the mice were intraperitoneally injected with $1 \mathrm{mg}$ Brdu and followed with infusion of Brdu using 7-day osmotic minipump (Alzet, Cupertino, CA, USA) at a rate of $60 \mu \mathrm{g} /$ day for 7 days. The mice were killed and perfused with $4 \%$ PFA after killing and femoral artery was dissected. The femoral artery was embedding in paraffin and $5-\mu \mathrm{m}$ sections were cut. After blocking and antigen retrieval, the slides were incubated with indicated antibodies. BrdU was stained with a 5-Bromo-2'-deoxy-Uridine Labeling and Detection Kit I (Roche, Indianapolis, IN, USA) following the kit instruction. Anti-SM22 $\alpha$ antibody was obtained from Abcam (catalogue number: ab10135) with 1:200 dilution. Second antibody was DyLight 549 Streptavidin (Vector laboratories, Burlingame, CA, USA).

Elastin staining. Elastin staining was performed using the Elastic stain kit from Sigma Aldrich (St. Louis, MO, USA). Briefly, deparaffinized slides were stained in Elastic stain solution for $10 \mathrm{~min}$. Then the slides were differentiated in working ferric chloride solution for $3 \mathrm{~min}$. After rinsing in 95\% alcohol, the slides were stained with Van Gieson solution for $3 \mathrm{~min}$. Then the slides were rinsed in $95 \%$ alcohol and dehydrated to xylene. The images were taken by Olympus FSX100 imaging system and were analyzed by Image J.

EDU incorporation. EDU incorporation was measured with an EDU flowcytometry kit. The cells were starved in the DMEM containg $0.1 \%$ BSA for $48 \mathrm{~h}$. Then the cells were stimulated with insulin or IGF-1. After $16 \mathrm{~h}$ of stimulation, the cells were incubated with $10 \mathrm{nM}$ EDU (Invitrogen, Carlsbad, CA, USA) for $4 \mathrm{~h}$. 
Table 1 Antibodies information

\begin{tabular}{|c|c|c|c|}
\hline Antibodies & Catalogue number & Source & Concentration \\
\hline $\operatorname{IR} \beta$ & $3020 s$ & Cell Signaling Technology & 1:1000 dilution \\
\hline Actin & sc-47778 HRP & Santa Cruz Biotechnology & 1:100,000 dilution \\
\hline IGF1R $\beta$ & 3027 & Cell Signaling Technology & 1:1000 dilution \\
\hline$p-A k t$ & 4060 & Cell Signaling Technology & 1:1000 dilution \\
\hline Akt & 9272 & Cell Signaling Technology & 1:1000 dilution \\
\hline GAPDH & ab9484 & Abcam & 1:2000 dilution \\
\hline p-IGF1R (Tyr 1135/1136)/IR (tyr 1150/1151) & 2969 & Cell Signaling Technology & 1:1000 dilution \\
\hline p-Erk & 9101 & Cell Signaling Technology & 1:1000 dilution \\
\hline Erk & 4695 & Cell Signaling Technology & 1:1000 dilution \\
\hline Pan-cadherin & sc-10733 & Santa Cruz Biotechnology & 1:1000 dilution \\
\hline Has2 (C-5) & sc-365263 & Santa Cruz Biotechnology & 1:500 dilution \\
\hline Anti-mouse IgG & sc-516102 & Santa Cruz Biotechnology & 1:5000 dilution \\
\hline Anti-rabbit IgG & sc-2357 & Santa Cruz Biotechnology & 1:5000 dilution \\
\hline
\end{tabular}

Then the cells were harvested and fixed with fixation buffer. After washing and permeabilizing with permeabilization buffer, the cells were incubated with Click$\mathrm{iT}^{\oplus}$ EdU reaction cocktail for $30 \mathrm{~min}$. After washing, the EDU-positive cells were measured by flow cytometer (BD LSR II). For 4-MU treatment, after starving with DMEM containg $0.1 \%$ BSA for $48 \mathrm{~h}$, the cells were incubated with $1 \mathrm{mM} 4-\mathrm{MU}$ for $4 \mathrm{~h}$. Then the medium was replaced with new DMEM containing $1 \mathrm{mM} 4-\mathrm{MU}$.

RNA sequencing. VSMCs were cultured from aortas of IGF1Rflox/flox mice or SMIGF1RKO mice. VSMCs from SMIGF1RKO mice were infected with AdCre to further knockdown IGF1R. VSMCs from IGF1Rflox/flox mice were infected with AdGFP. The VSMCs were starved with $0.1 \%$ BSA for $48 \mathrm{~h}$ and then stimulated with insulin for $4 \mathrm{~h}$. RNA was extracted with the Purelink RNA mini kit (The life technologies, Carsbad, CA, USA) and 100 ng of RNA was used to create library for RNA sequencing ( $n=3$ for each group). RNA sequencing was performed using Illumina NextSeq 500- Single Read 75 (SR75) by the Center for Cancer Computational Biology (CCCB) at Dana Farber Cancer Institute of Harvard Medical School. Sequencing reads from FASTQ files were aligned to the reference genome using the RNA-specific STAR aligner to generate sequence alignment (BAM) files. The featureCounts tool was then used taking in the BAM files to count sequencing reads mapping to the reference genome at the gene level. The read counts were subsequently normalized between samples using DESeq. The normalized readcount file was further used in RNA-Seq analysis. RNA sequencing data were analyzed with limma, an $\mathrm{R}$ package for linear modeling that powers differential expression analyses. We controlled batch effects and used sample quality weights.

Real-time PCR. One microgram of mRNA was used to generate cDNA using the high-capacity cDNA reverse transcription kit (Applied Biosystems, Grand Island, NY, USA). Gene expression level was normalized to the expression level of 36B4.

The primer sequences are listed in Table 2.

Insulin and IGF-1 binding. Cells were incubated with $\mathrm{I}^{125}$-labeled insulin or IGF-1 (PerkinElmer, Waltham, MA, USA) with indicated concentration of unlabeled insulin or IGF-1 on ice for $5 \mathrm{~h}$. After washing with cold PBS for three times, the cells were lysed with $4 \% \mathrm{NaOH}$ for $1 \mathrm{~h}$ at room temperature. Then the cell lysis was transferred to the scintillation bottle, and the radioactivity was measured by a scintillation counter.

Generation of adenovirus containing IR-ECD/IGF1R-ICD or IGF1R-ECD/IR-ICD chimeric receptor. Plasmids containing IR-ECD/IGF1R-ICD or IGF1R-ECD/IRICD chimeric receptor were kindly provided by Dr. C. Ronald Kahn (Joslin Diabetes Center, Boston, MA, USA). The chimeric receptors were cloned into adenovirus backbone plasmid using ViraPower Adenoviral Gateway Expression kit (Invitrogen, Carlsbad, CA, USA). The VSMCs were infected with adenovirus at $100 \mathrm{MOI}$

Measurement of hyaluronan in medium. The concentration of hyaluronan in cell cultured medium was determined by the hyaluronan ELISA kit (R\&D systems, Minneapolis, MN, USA).

Hyaluronan staining. The sections were incubated with biotin-conjugated hyaluronic acid-binding protein $(8 \mu \mathrm{g} / \mathrm{ml}$, Fisher, Waltham, MA, USA $)$ at $4{ }^{\circ} \mathrm{C}$ overnight. After washing, the slides were incubated with avidin-conjugated dylight 549 .

SiRNA transfection. Control siRNA (The Silencer Select Negative Control No. 1 siRNA) or HAS2 siRNA (\#s67368, sense, 5'-GACCUACCCUGGGAUUAAAtt$3^{\prime}$; antisense, 5'-UUUAAUCCCAGGGUAGGUCag- $3^{\prime}$ ) were predesigned by Ambion (Foster City, CA, USA). $10 \mathrm{nM}$ siRNAs were transfected to VSMCs using
Table 2 Primer information

$\begin{array}{ll}\text { 36b4 UP } & \text { CCGGATGTGAGGCAGCAG } \\ \text { 36b4 DN } & \text { GCTCCAAGCAGATGCAGCA } \\ \text { IR UP } & \text { GGAAGTACATCTGATTCGAGG } \\ \text { IR DN } & \text { TGAGTGATGGTGAGGTTGT } \\ \text { Egln3 UP } & \text { GAAGGAAATCGTTTTAGCAG } \\ \text { EgIn3 DN } & \text { GAAGTACCAGACAGTCATAGCG } \\ \text { Sipr3 UP } & \text { AGAGTGTCATTTCCCAAGACTG } \\ \text { Sipr3 DN } & \text { ACTCTCCCAATTGTTCCCTG } \\ \text { Slc16a3 UP } & \text { CCTGTCATGCTTGTGGGT } \\ \text { Slc16a3 DN } & \text { GGAAGGCTGGAAGTTAGAG } \\ \text { Rcor2 UP } & \text { GCATGTACCTGAGTCCTGAAG } \\ \text { Rcor2 DN } & \text { CTGCTATTGGTCTGCTTCATG } \\ \text { Adamts14 UP } & \text { AACTCTTCCGACAACCCTTG } \\ \text { Adamts14 DN } & \text { GTAGTCTGAGTTGTCTGTGCG } \\ \text { PfkI UP } & \text { GGAAAGCTATCTCATCCAGC } \\ \text { PfkI DN } & \text { CCATACCCATCTTGCTACTCA } \\ \text { Pfkfb3 UP } & \text { CTGACTCGCTACCTCAACTG } \\ \text { Pfkfb3 DN } & \text { ACTGTTTTCGGACTCTCATGG } \\ \text { Relt UP } & \text { CCCTGTCTTGCCTCTTTGG } \\ \text { Relt DN } & \text { CATAATGTGCCCTGTCCTGG } \\ \text { Gys1 UP } & \text { CCATGTCTTCACTACCGTATCC } \\ \text { Gys1 DN } & \text { CTCTGAGCATGAAGGTTCTGG } \\ \text { Has2 UP } & \text { AGTCATGTACACAGCCTTCAG } \\ \text { Has2 DN } & \text { CTCCAACACCTCCAACCATAG } \\ \text { Kif26b UP } & \text { TCCCACAAGACGCTTCTCAG } \\ \text { Kif26b DN } & \text { TCGTCCCTTCCGATCATGGTG } \\ \text { Trabd2b UP } & \text { GATGAAGATGTGCTGAGAGGG } \\ \text { Trabd2b DN } & \text { CTAGGGATGTGGAAGTGTCTC } \\ \text { IGF1R UP } & \text { GTACCGCATCGATATCCACAG } \\ \text { IGF1R DN } & \text { ATGGAGTTTTCGGGTCTT } \\ & \end{array}$

Lipofectamine RNAiMAX from Invitrogen (Carlsbad, CA, USA) according to the manufacturer's instructions. Knockdown efficiency was confirmed after $24 \mathrm{~h}$ and $72 \mathrm{~h}$ transfection with siRNAs.

Statistics. Measurements are from distinct samples. Comparisons of the two groups were made using paired or unpaired $t$ test as appropriate. Comparison among more than two groups was performed by one-way ANOVA or two-way ANOVA followed by the post hoc analysis to evaluate statistical significance between the two groups. Statistical significance was defined as $p<0.05$. In text and graphs, the data are presented as the mean \pm standard error.

Reporting summary. Further information on research design is available in the Nature Research Reporting Summary linked to this article.

\section{Data availability}

The data sets generated during and/or analyzed during this study are available from the corresponding author on reasonable request. The source data underlying Figs. 1-7 and Supplementary Figs. 1-14 are provided as a Source Data file. The RNA sequencing data 
set (normalized counts) is provided as a Supplementary Dataset in the excel format. The BAM or FASTQ files were not saved before the closing of the core at DFCI, hence the limitations to the accessibility of the raw data.

Received: 20 June 2018; Accepted: 3 September 2019;

Published online: 27 September 2019

\section{References}

1. Byrne, R. A., Stone, G. W., Ormiston, J. \& Kastrati, A. Coronary balloon angioplasty, stents, and scaffolds. Lancet 390, 781-792 (2017).

2. Kedhi, E. et al. Impact of coronary lesion complexity on drug-eluting stent outcomes in patients with and without diabetes mellitus: analysis from 18 pooled randomized trials. J. Am. Coll. Cardiol. 63, 2111-2118 (2014).

3. Casscells, W. Migration of smooth muscle and endothelial cells. Critical events in restenosis. Circulation 86, 723-729 (1992).

4. Virmani, R. \& Farb, A. Pathology of in-stent restenosis. Curr. Opin. Lipidol. 10, 499-506 (1999).

5. King, G. L. et al. Differential responsiveness to insulin of endothelial and support cells from micro- and macrovessels. J. Clin. Investig. 71, 974-979 (1983).

6. King, G. L., Goodman, A. D., Buzney, S., Moses, A. \& Kahn, C. R. Receptors and growth-promoting effects of insulin and insulinlike growth factors on cells from bovine retinal capillaries and aorta. J. Clin. Investig. 75, 1028-1036 (1985).

7. Du, J. \& Delafontaine, P. Inhibition of vascular smooth muscle cell growth through antisense transcription of a rat insulin-like growth factor I receptor cDNA. Circulation Res. 76, 963-972 (1995).

8. Pfeifle, B. \& Ditschuneit, H. Effect of insulin on growth of cultured human arterial smooth muscle cells. Diabetologia 20, 155-158 (1981).

9. Bornfeldt, K. E. et al. Insulin-like growth factor-I and platelet-derived growth factor-BB induce directed migration of human arterial smooth muscle cells via signaling pathways that are distinct from those of proliferation. J. Clin. Investig. 93, 1266-1274 (1994).

10. Bornfeldt, K. E., Arnqvist, H. J. \& Capron, L. In vivo proliferation of rat vascular smooth muscle in relation to diabetes mellitus insulin-like growth factor I and insulin. Diabetologia 35, 104-108 (1992).

11. Aneke-Nash, C. S. et al. Changes in insulin-like growth factor-I and its binding proteins are associated with diabetes mellitus in older adults. J. Am. Geriatrics Soc. 63, 902-909 (2015).

12. Indolfi, C. et al. Effects of balloon injury on neointimal hyperplasia in streptozotocin-induced diabetes and in hyperinsulinemic nondiabetic pancreatic islet-transplanted rats. Circulation 103, 2980-2986 (2001).

13. Park, S. H. et al. Neointimal hyperplasia after arterial injury is increased in a rat model of non-insulin-dependent diabetes mellitus. Circulation 104, 815-819 (2001).

14. Qi, W. et al. SHP-1 activation inhibits vascular smooth muscle cell proliferation and intimal hyperplasia in a rodent model of insulin resistance and diabetes. Diabetologia 60, 585-596 (2017).

15. King, G. L., Park, K. \& Li, Q. Selective insulin resistance and the development of cardiovascular diseases in diabetes: The 2015 Edwin Bierman Award Lecture. Diabetes 65, 1462-1471 (2016).

16. De Meyts, P. \& Whittaker, J. Structural biology of insulin and IGF1 receptors: implications for drug design. Nat. Rev. Drug Discov. 1, 769-783 (2002).

17. Kahn, C. R., Freychet, P., Roth, J. \& Neville, D. M. Jr. Quantitative aspects of the insulin-receptor interaction in liver plasma membranes. J. Biol. Chem. 249, 2249-2257 (1974).

18. Boucher, J., Kleinridders, A. \& Kahn, C. R. Insulin receptor signaling in normal and insulin-resistant states. Cold Spring Harb. Perspect. Biol. 6, a009191 (2014).

19. Kitamura, T., Kahn, C. R. \& Accili, D. Insulin receptor knockout mice. Annu. Rev. Physiol. 65, 313-332 (2003).

20. Kadowaki, T. et al. Five mutant alleles of the insulin receptor gene in patients with genetic forms of insulin resistance. J. Clin. Investig. 86, 254-264 (1990).

21. Accili, D. et al. Early neonatal death in mice homozygous for a null allele of the insulin receptor gene. Nat. Genet. 12, 106-109 (1996).

22. Joshi, R. L. et al. Targeted disruption of the insulin receptor gene in the mouse results in neonatal lethality. EMBO J. 15, 1542-1547 (1996).

23. Steele-Perkins, G. et al. Expression and characterization of a functional human insulin-like growth factor I receptor. J. Biol. Chem. 263, 11486-11492 (1988).

24. Lammers, R., Gray, A., Schlessinger, J. \& Ullrich, A. Differential signalling potential of insulin- and IGF-1-receptor cytoplasmic domains. EMBO J. 8, 1369-1375 (1989).

25. Abuzzahab, M. J. et al. IGF-I receptor mutations resulting in intrauterine and postnatal growth retardation. New Engl. J. Med. 349, 2211-2222 (2003).

26. Liu, J. P., Baker, J., Perkins, A. S., Robertson, E. J. \& Efstratiadis, A. Mice carrying null mutations of the genes encoding insulin-like growth factor I (Igf1) and type 1 IGF receptor (Igf1r). Cell 75, 59-72 (1993).
27. Bright, G. M. Recombinant IGF-I: past, present and future. Growth Horm. IGF Res. 28, 62-65 (2016)

28. Ebina, Y. et al. The human insulin receptor cDNA: the structural basis for hormone-activated transmembrane signalling. Cell 40, 747-758 (1985).

29. Ullrich, A. et al. Insulin-like growth factor I receptor primary structure: comparison with insulin receptor suggests structural determinants that define functional specificity. EMBO J. 5, 2503-2512 (1986).

30. Whittaker, J., Soos, M. A. \& Siddle, K. Hybrid insulin receptors. Molecular mechanisms of negative-dominant mutations in receptor-mediated insulin resistance. Diabetes Care 13, 576-581 (1990).

31. Liu, S. \& Cheng, C. Akt signaling is sustained by a CD44 splice isoformmediated positive feedback loop. Cancer Res. 77, 3791-3801 (2017).

32. Dharaneeswaran, H. et al. FOXO1-mediated activation of Akt plays a critical role in vascular homeostasis. Circ. Res. 115, 238-251 (2014).

33. Bayes-Genis, A., Conover, C. A. \& Schwartz, R. S. The insulin-like growth factor axis: a review of atherosclerosis and restenosis. Circulation Res. 86, 125-130 (2000).

34. Folli, F., Ghidella, S., Bonfanti, L., Kahn, C. R. \& Merighi, A. The early intracellular signaling pathway for the insulin/insulin-like growth factor receptor family in the mammalian central nervous system. Mol. Neurobiol. 13 , 155-183 (1996).

35. Ulanet, D. B., Ludwig, D. L., Kahn, C. R. \& Hanahan, D. Insulin receptor functionally enhances multistage tumor progression and conveys intrinsic resistance to IGF-1R targeted therapy. Proc. Natl Acad. Sci. USA 107, 10791-10798 (2010).

36. Cai, W. et al. Domain-dependent effects of insulin and IGF-1 receptors on signalling and gene expression. Nat. Commun. 8, 14892 (2017).

37. Kiselyov, V. V., Versteyhe, S., Gauguin, L. \& De Meyts, P. Harmonic oscillator model of the insulin and IGF1 receptors' allosteric binding and activation. Mol. Syst. Biol. 5, 243 (2009).

38. Schumacher, R., Mosthaf, L., Schlessinger, J., Brandenburg, D. \& Ullrich, A. Insulin and insulin-like growth factor-1 binding specificity is determined by distinct regions of their cognate receptors. J. Biol. Chem. 266, 19288-19295 (1991).

39. Abbas, A. et al. The insulin-like growth factor-1 receptor is a negative regulator of nitric oxide bioavailability and insulin sensitivity in the endothelium. Diabetes 60, 2169-2178 (2011).

40. Evanko, S. P., Angello, J. C. \& Wight, T. N. Formation of hyaluronan- and versican-rich pericellular matrix is required for proliferation and migration of vascular smooth muscle cells. Arteriosclerosis, Thrombosis, Vasc. Biol. 19, 1004-1013 (1999).

41. Savani, R. C. et al. Migration of bovine aortic smooth muscle cells after wounding injury. The role of hyaluronan and RHAMM. J. Clin. Investig. 95, 1158-1168 (1995).

42. Simpson, R. M. et al. Hyaluronan is crucial for stem cell differentiation into smooth muscle lineage. Stem Cells 34, 1225-1238 (2016).

43. Camenisch, T. D. et al. Disruption of hyaluronan synthase-2 abrogates normal cardiac morphogenesis and hyaluronan-mediated transformation of epithelium to mesenchyme. J. Clin. Investig. 106, 349-360 (2000).

44. Riessen, R., Wight, T. N., Pastore, C., Henley, C. \& Isner, J. M. Distribution of hyaluronan during extracellular matrix remodeling in human restenotic arteries and balloon-injured rat carotid arteries. Circulation 93, 1141-1147 (1996).

45. Kashima, Y. et al. Crucial role of hyaluronan in neointimal formation after vascular injury. PloS One 8, e58760 (2013).

46. Chung, S. A. et al. Pirfenidone attenuates the IL-1beta-induced hyaluronic acid increase in orbital fibroblasts from patients with thyroid-associated ophthalmopathy. Investigative Ophthalmol. Vis. Sci. 55, 2276-2283 (2014).

\section{Acknowledgements}

This study was supported by NIH grant R01-DK-053105. We are grateful for the expert technical assistance provided by Biostatistic and Bioinformatic Core, Advanced Microscopy Core, Flow Cytometry Core, Mouse Physiology Core, Genomic Core, which are supported by NIH grants 5P30DK036836 and S10OD021740. We thank Dr. Kevin Croce for the technique support in femoral artery wire injury. The content of this paper is solely the responsibility of the authors, and does not necessarily represent the official views of the funding agencies.

\section{Author contributions}

Q.L. performed experiments, analyzed the data, and wrote the paper. J.F., Y.X., W.Q., Q.H. and W.C. performed experiments. A.I, K.P., J.F., H.Y., C.R.K. and C.R.M. participated in the discussions and critical review of the paper; G.L.K. supervised the studies and wrote the paper.

\section{Competing interests}

The authors declare no competing interests. 


\section{Additional information}

Supplementary Information is available for this paper at https://doi.org/10.1038/ s41467-019-12368-2.

Correspondence and requests for materials should be addressed to G.L.K.

Peer review information Nature Communications thanks the anonymous reviewers for their contribution to the peer review of this work. Peer reviewer reports are available.

Reprints and permission information is available at http://www.nature.com/reprints

Publisher's note Springer Nature remains neutral with regard to jurisdictional claims in published maps and institutional affiliations. (c) (i) Open Access This article is licensed under a Creative Commons Attribution 4.0 International License, which permits use, sharing, adaptation, distribution and reproduction in any medium or format, as long as you give appropriate credit to the original author(s) and the source, provide a link to the Creative Commons license, and indicate if changes were made. The images or other third party material in this article are included in the article's Creative Commons license, unless indicated otherwise in a credit line to the material. If material is not included in the article's Creative Commons license and your intended use is not permitted by statutory regulation or exceeds the permitted use, you will need to obtain permission directly from the copyright holder. To view a copy of this license, visit http://creativecommons.org/ licenses/by/4.0/.

(C) The Author(s) 2019 